\title{
Forced vibration and low-velocity impact of laminated composite plates $^{\dagger}$
}

\author{
ASGHAR NOSIER ${ }^{\dagger}$, RAKESH K KAPANIA ${ }^{\ddagger}$ and J N REDDY* \\ Aerospace \& Ocean Engineering Department, Virginia Polytechnic \\ Institute and State University, Blacksburg, VA 24061, USA \\ * Present address: Oscar S Wyatt Chair, Department of Mechanical \\ Engineering, Texas A \& M University, College Station, TX 77843, USA
}

\begin{abstract}
The layerwise theory of Reddy is used to study the low velocity impact response of laminated plates. Forced-vibration analysis is developed by the modal superposition technique. Six different models are introduced for representation of the impact pressure distribution. The first five models, in which the contact area is assumed to be known, result in a nonlinear integral equation similar to the one obtained by Timoshenko in 1913. The resulting nonlinear integral equation is discretised using a time-element scheme. Two different interpolation functions, namely: (i) Lagrangian and (ii) Hermite, are used to express the impact force. The Hermitian polynomialbased representation, obviously more sophisticated, is introduced to verify the Lagrangian-based representation. Due to its modular nature the present numerical technique is preferable to the existing numerical methods in the literature. The final loading model, in which the time dependence of the contact area is taken into account according to the Hertzian contact law, resulted in a relatively more complicated but more realistic, nonlinear integral equation. The analytical developments concerning this model are all new and are reported for the first time in this paper. Also a simple, but accurate, numerical technique is developed for solving our new nonlinear integral equation which results in the timehistory of the impact force. Our numerical results are first tested with a series of existing example problems. Then a detailed study concerning all the response quantities, including the in-plane and interlaminar stresses, is carried out for symmetric and antisymmetric cross-ply laminates and important conclusions are reached concerning the usefulness and accuracy of the various plate theories.
\end{abstract}

Keywords. Low-velocity impact; laminated composites; layerwise theory; forced vibration; nonlinear integral equation.

\footnotetext{
${ }^{+}$This paper, presented at a symposium to celebrate the Golden Jubilee of the Aerospace Department, Indian Institute of Science, Bangalore first appeared as a paper in Computational Mechanics (1994) 13: 360-379 (C) Springer-Verlag. Reproduced here with slight alterations with permission.
} 


\section{Introduction}

Composites are often used in situations involving the sudden application of loads. The dynamic response of the structure ensues after load application and a state of stress leading to failure may be generated. It is necessary to understand the response characteristics of the material body for all important effects, including geometry, boundary conditions, and loading.

One of the major obstacles that prevents application of these materials in primary structures is the damage induced due to service or accidental loads (e.g., bird impact), and the consequent reduction in stiffness, strength and life of these structures. Therefore, damage-resistant and durable composite materials are essential for the design of lighter and easier-to-maintain structures. The composite laminate is a fundamental building block of a composite structure. Hence, an understanding of damage development and failure behaviour in a composite laminate is a basic requirement for understanding the failure behaviour of a composite structure. Investigations in the area of wave propagation in laminated media have been conducted by geologists and physicists interested in the study of the wave propagation of seismic waves. The increasing use of laminated composites in aerospace, automotive and naval structures has led to a more elaborate area of research. These structures are subject to high velocity impact by birds, meteoroids, and undersea animals. Detailed reviews of some of these studies were given by Kapania \& Raciti (1989) and Abrate (1991). Only a brief review of some of the pertinent recent analytical studies is presented in the following pages.

The transient response of laminated plates subjected to impact loads was investigated by analytical and numerical methods. Goldsmith (1960) used the normal modes method to determine the dynamic response of an isotropic plate or beam to a rigid impactor. Timoshenko (1913) used normal modes and a Hertzian contact law to analyse the deflections of a beam due to impact. The resulting nonlinear integral equations were solved by numerical integration. Sun \& Chattopadhyay (1975) extended Timoshenko's method to a laminated simply-supported composite plate. Ramkumar \& Chen (1983) used the Fourier integral transform to find the response of an infinite anisotropic laminated plate to an experimentally determined impact force. Petersen (1985) used the finite method based on a shear deformable plate theory with rotary inertia to analyse laminated plates subjected to impact loads. Thangitham et al (1987) obtained low-velocity impact responses of orthotropic plates using a higher-order theory that incorporates: (i) the transverse normal stress, (ii) rotary inertia effects, and (iii) fulfills the shear stress boundary conditions on the bounding surfaces. Sun \& Liou (1989) used a three-dimensional hybrid stress finite element method to investigate laminated plates under impact loads. Cairns \& Lagace (1989) obtained transient response of graphite/epoxy and kevlar/epoxy laminates subjected to impact using the Rayleigh-Ritz method.

For the study of impact response of metals and composites, many researchers used the Hertzian contact law, which relates impactor and plate motion with contact force. However, Yang \& Sun (1981) showed that the Hertzian contact law was not adequate by performing statical indentation tests on graphite/epoxy composite laminates using spherical steel indentors of different sizes. They found that significant permanent indentations existed. In order to account for the permanent indentation, Tan \& Sun (1982) proposed a modified Hertzian contact law following Yang \& Sun (1981). They compared experimental results with the predictions of finite element analysis using the statically determined contact law. Sun $\&$ Chen (1985) analysed initially stressed 
composite plates under impact loads using this modified Hertzian contact law. Bogdanovich \& Yarve (1989) proposed a method which combined the calculation of stress-strain states in a laminated plate on the basis of spline-approximation of displacements. A variational approach was used for studying the process of impact contact interaction between the indentor and the plate. This method, capable of taking into account the high-velocity transverse (through-the-thickness) stress waves was subsequently extended to the calculation of damage zones in laminated composite plates subjected to low velocity impact (Bogdanovich \& Yarve 1990). Sankar \& Sun (1985b) used plane stress finite elements to study the low-velocity impact of laminated beams subjected to initial stresses.

Most of the impact problems have been formulated using the small deflection theory, which is adequate if the impact load is small. However, if a plate undergoes large deflections of the order of the thickness of the plate, it is necessary to include the geometric nonlinearity. Chen \& Sun (1985) investigated the nonlinear transient response of composite laminates subjected to impact loads with initial stresses. They used the finite element method based on the Mindlin plate theory in conjunction with an experimentally established contact law (Tan \& Sun 1982). Kant \& Mallikarjuna (1991) used a higher-order theory and $C^{0}$ finite elements to analyse a laminated plate under impact loads using the modified Hertzian contact law by Tan \& Sun (1982). Obst \& Kapania (1992) studied the geometrically nonlinear impact response of laminated beams using a third-order shear deformation theory. These authors also considered the effect of initial stresses. Effect of geometric imperfections on the geometrically nonlinear impact response of thin laminated plates was studied by Byun \& Kapania (1992).

It is important to predict the combined effect of various damage modes and the external environment on the degradation and failure of the laminate. However, it appears that not much work has been done to understand and quantify the combined effect of these damage modes on the performance of composite laminates, whereas this understanding is essential for efficient design and production of damage resistant and durable laminated composite structures under impact loads. Hence, there is a need for developing a general tool to predict the combined effect of various damage modes on the performance of the composite structures with complex geometry and loading conditions.

To study the impact-generated damage, it is important to get very accurate information for in-plane and transverse stresses. However, finite element models based on the classical laminated plate theory (CLPT) or the first order shear deformation theory (FSDPT) cannot give accurate interlaminar stresses from constitutive relations. The equilibrium equations of 3-D elasticity give accurate interlaminar stresses. A postprocessor for transverse normal and shear stresses as well as in-plane stresses was developed by Byun \& Kapania (1991) using the finite element codes employing the CLPT and first order shear deformation theory. The transverse stresses were obtained by integrating the 3-D elasticity equilibrium equations. The postprocessor uses global interpolation of the nodal displacements obtained from the finite element analysis. Using a finite elements program based on both CLPT (Kapania \& Yang 1986) and FSDPT (Reddy 1980), the transverse stresses were obtained for both symmetrically and unsymmetrically laminated plates. Good agreement with the 3-D elasticity results given by Pagano (1969) for symmetrically laminated plates and by Chaudhuri \& Seide (1987) for unsymmetrically laminated plates was obtained. This formulation did not include the inertia effects.

An alternate way to accurately calculate the in-plane and transverse stresses, 
without directly integrating the equilibrium equations, is to use Reddy's layerwise theory (Reddy 1987). One of the objectives of the present work is to use this theory for studying the impact response of laminated plates and to compare its performance to that of Reddy's first-order (Reddy 1983) and third-order (Reddy 1990) shear deformation theories. The layerwise theory of Reddy is expected to be more efficient than performing a conventional three-dimensional finite element analysis of impact response.

Recent experimental studies by Qian \& Swanson (1990) and Swanson et al (1991) have shown that to accurately calculate the stresses in the vicinity of the impact, it is important to account for the distribution of the impact load. Note that it is very important that the stresses under the impactor be obtained very accurately as the matrix failure may initiate under the point of impact (Pintado et al 1991). In the present study, six different distributions, labelled as cases I through VI (with varying complexity), were conceived and the expressions for the generalized forces (to be used in the modal superposition technique) corresponding to each distribution were derived.

The first three models, in which the impact load is presented as (i) a concentrated load (model I), (ii) a uniform load distributed over a rectangular contact region (model II), and (iii) a sinusoidally distributed load (model III) over a rectangular contact region, are already considered in the literature. In model IV the load is assumed to be uniformly distributed over a circular region. This model is implemented in finite element codes by various researchers. Here, however, we considered it in our analytical solutions. In model $\mathrm{V}$ the impact load is assumed to be spherically distributed over a circular region in accordance with the Hertzian contact law. The analytical expression for the generalized forces corresponding to this load is also obtained for the first time in this paper. Despite the fact that the size of the contact region varies with time, it is assumed that the size of the contact region is known in cases I through $\mathrm{V}$ loading models. For models I through $\mathrm{V}$, we obtain a nonlinear integral equation, whose solution yields time histories of the impact force, displacement, and other response quantities. Finally in model VI we incorporated certain relations, from the Hertzian contact law, into the case $V$ loading model and obtained a slightly different nonlinear integral equation in which the time variance of the contact region is taken into account.

An iterative scheme with a small time increment is often used to obtain the response time-histories. Sankar \& Sun (1985a) developed a numerical scheme in which the impact force is represented by a series of ramp functions. These ramp functions increase linearly from zero to unity during a time step and retain this value for the subsequent duration. Using a series of these ramp functions, one can calculate the value of the dynamic Green's function for a given structure. The advantage of this approach was that the iterations for solving the nonlinear equation for calculating the impact force were separated from the structural response calculations. In the present study, a different approach is proposed. The impact force is assumed to be represented by a series of piecewise basis functions (finite elements) in time. As a first step, the impact force is represented as a series of piecewise linear Lagrange interpolation functions (the so-called hat functions). An obvious advantage of this approach is its modularity, i.e., one can easily replace the linear interpolating polynomials by higher-order functions. Indeed, in this study we have also represented the impact force by using the time-finite-elements using cubic Hermite interpolating polynomials with minimal changes in the overall analysis. 


\section{Forced-vibration analysis: General results}

Owing to the complex nature of dynamic load transfer characteristics, the response analysis of an impacted plate is more complicated than an ordinary dynamic problem in which the distribution of the transverse load is known. That is, the solution of a nonlinear integral equation is necessitated which, on the other hand, will result in the simultaneous knowledge of impact force and response quantities of a plate. For most part, however, the usual procedures used in an ordinary response analysis calculations can be utilised to determine the general analytical expressions for the response quantities in terms of the unknown impact force.

The systems of the differential eigenvalue equations of a laminated plate using the layerwise theory, presented in a report (Nosier et al 1992), can be shown to be selfadjoint. Therefore, we can make a positive statement about the orthogonality of the eigenfunctions, provided the eigenfrequencies (i.e. natural frequencies) are distinct. As a result, modal analysis can be conveniently used to obtain the response.

\subsection{Orthogonality relationships of normal modes}

The orthogonality relationships in various plate theories can be obtained by the use of standard procedures in the analysis of continuous systems. In the layerwise plate theory (LWPT), for example, if we let $U_{m n k}^{j}, V_{m n k}^{j}$, and $W_{m n k}^{j}$ (see Nosier et al 1992) and $U_{p q r}^{i}, V_{p q r}^{i}$, and $W_{p q r}^{i}$ denote the eigenfunctions corresponding to distinct eigenfrequencies $\omega_{m n k}$ and $\omega_{p q r}$, respectively, the orthogonality relation can be stated as:

$$
\begin{array}{r}
\int_{0}^{b} \int_{0}^{a}\left[I^{i j}\left(U_{m n k}^{j} U_{p q r}^{i}+V_{m n k}^{j} V_{p q r}^{i}+W_{m n k}^{j} W_{p q r}^{i}\right)\right] \mathrm{d} x \mathrm{~d} y \\
= \begin{cases}0, & \text { if } m \neq p \text { and/or } n \neq q \text { and/or } k \neq r, \\
N_{m n k}, & \text { if } m=p, n=q, \text { and } k=r .\end{cases}
\end{array}
$$

Similarly, in third-order shear deformation theory (TSDPT, $\lambda=1$ ) and first-order shear deformation theory (FSDPT, $\lambda=0$ ) we have:

$$
\begin{aligned}
\int_{0}^{b} \int_{0}^{a} & I_{1}\left(U_{m n k} U_{p q r}+V_{m n k} V_{p q r}+W_{m n k} W_{p q r}\right) \\
& +\bar{I}_{2}\left(U_{m n k} \Psi_{x_{p q r}}+\Psi_{x_{m n k}} U_{p q r}+V_{m n k} \Psi_{x_{p q r}}+\Psi_{y_{m n k}} V_{p q r}\right) \\
& +\bar{I}_{3}\left(\Psi_{x_{m n k}} \Psi_{x_{p q r}}+\Psi_{y_{m n k}} \Psi_{y_{p q r}}\right) \\
& -\lambda \frac{4}{3 h^{2}} I_{4}\left(U_{m n k} W_{p q r, x}+W_{m n k, x} U_{p q r}+V_{m n k} W_{p q r, y}+W_{m n k, y} V_{p q r}\right) \\
& -\lambda \frac{4}{3 h^{2}} \bar{I}_{5}\left(\Psi_{x_{m n n k}} W_{p q r, x}+W_{m n k, x} \Psi_{x_{p q r}}+\Psi_{y_{m n k}} W_{p q r, x}+W_{m n k, y} \Psi_{y_{p q r}}\right) \\
& \left.+\lambda\left(\frac{4}{3 h^{2}}\right)^{2} I_{7}\left(W_{m n k, x} W_{p q r, x}+W_{m n k, y} W_{p q r, y}\right)\right] \mathrm{d} x \mathrm{~d} y \\
& = \begin{cases}0, & \text { if } m \neq p \text { and } / \text { or } n \neq q \text { and } / \text { or } k \neq r \\
N_{m n k}, & \text { if } m=p, n=q, \text { and } k=r\end{cases}
\end{aligned}
$$


and in CLPT

$$
\begin{gathered}
\int_{0}^{b} \int_{0}^{a} I_{1}\left(U_{m n k} U_{p q r}+V_{m n k} V_{p q r}+W_{m n k} W_{p q r}\right) \mathrm{d} x \mathrm{~d} y \\
\quad= \begin{cases}0, & \text { if } m \neq p \text { and/or } n \neq q \text { and } / \text { or } k \neq r, \\
N_{m n k}, & \text { if } m=p, n=q, \text { and } k=r .\end{cases}
\end{gathered}
$$

For a simply-supported plate the generalized masses $N_{m n k}$ in various theories are given as (see Nosier et al 1992):

LWPT

$$
N_{m n k}=(a b / 4) I^{i j}\left(A_{m n k}^{i} A_{m n k}^{j}+B_{m n k}^{i} B_{m n k}^{j}+C_{m n k}^{i} C_{m n k}^{j}\right) .
$$

$\operatorname{TSDPT}(\lambda=1), \operatorname{FSDPT}(\lambda=0)$

$$
\begin{aligned}
N_{m n k}=( & a b / 4)\left[I_{1}\left(A_{m n k}^{2}+B_{m n k}^{2}+E_{m n k}^{2}\right)+2 \bar{I}_{2}\left(A_{m n k} C_{m n k}+B_{m n k} D_{m n k}\right)\right. \\
& +\bar{I}_{3}\left(C_{m n k}^{2}+D_{m n k}^{2}\right)-\lambda\left(8 / 3 h^{2}\right) I_{4}\left(\alpha_{m} A_{m n k} E_{m n k}+\beta_{n} B_{m n k} E_{m n k}\right) \\
& \left.-\lambda\left(8 / 3 h^{2}\right) \bar{I}_{5}\left(\alpha_{m} C_{m n k}+\beta_{n} D_{m n k}\right) E_{m n k}+\lambda\left(4 / 3 h^{2}\right)^{2} I_{7}\left(\alpha_{m}^{2}+\beta_{n}^{2}\right) E_{m n k}^{2}\right]
\end{aligned}
$$

CLPT

$$
N_{m n k}=(a b / 4) I_{1}\left(A_{m n k}^{2}+B_{m n k}^{2}+E_{m n k}^{2}\right) \text {. }
$$

Note that a repeated index in (4) indicates summation of terms over the range of that index.

\subsection{Response analysis, general results}

As we pointed out earlier, the response of the laminate to transverse excitation can be obtained conveniently by modal analysis. To this end, we illustrate the analysis within LWPT and summarize the appropriate results of the equivalent single-layer theories. According to normal mode analysis, we expand the primary response quantities as

$$
\begin{aligned}
& u^{j}(x, y, t)=\sum_{m=1}^{\infty} \sum_{n=1}^{\infty} \sum_{k=1}^{3(N+1)} U_{m n k}^{j}(x, y) \cdot \zeta_{m n k}(t), \\
& v^{j}(x, y, t)=\sum_{m=1}^{\infty} \sum_{n=1}^{\infty} \sum_{k=1}^{3(N+1)} V_{m n k}^{j}(x, y) \cdot \zeta_{m n k}(t), \\
& w^{j}(x, y, t)=\sum_{m=1}^{\infty} \sum_{n=1}^{\infty} \sum_{k=1}^{3(N+1)} W_{m n k}^{j}(x, y) \cdot \zeta_{m n k}(t), j=1,2, \ldots, N+1,
\end{aligned}
$$

where $W_{m n k}^{j}, V_{m n k}^{j}$, and $W_{m n k}^{j}$ are the eigenfunctions and $\zeta_{m n k}$ are the time-dependent generalized coordinates, yet to be determined.

Upon substitution of (7) into the equations of motion we obtain (see Nosier et al 1992)

$$
\sum_{m=1}^{\infty} \sum_{n=1}^{\infty} \sum_{k=1}^{3(N+1)} I^{i j} U_{m n k}^{j}\left(\zeta_{m n k}+\omega_{m n k}^{2} \zeta_{m n k}\right)=0
$$




$$
\begin{aligned}
& \sum_{m=1}^{\infty} \sum_{n=1}^{\infty} \sum_{k=1}^{3(N+1)} I^{i j} V_{m n k}^{j}\left(\xi_{m n k}+\omega_{m n k}^{2} \zeta_{m n k}\right)=0 \\
& \sum_{m=1}^{\infty} \sum_{n=1}^{\infty} \sum_{k=1}^{3(N+1)} I^{i j} W_{m n k}^{j}\left(\xi_{m n k}+\omega_{m n k}^{2} \zeta_{m n k}\right)=\delta_{1} P_{z} .
\end{aligned}
$$

Now we multiply (8a), (8b), and (8c), respectively, by $U_{p q r}^{i}, V_{p q r}^{i}, W_{p q r}^{i}$ and add the results so obtained. We obtain

$$
\begin{aligned}
& \sum_{m=1}^{\infty} \sum_{n=1}^{\infty} \sum_{k=1}^{3(N+1)}\left(\zeta_{m n k}+\omega_{m n k}^{2} \zeta_{m n k}\right) \\
& \quad \times\left[I^{i j}\left(U_{m n k}^{j} U_{p q r}^{i}+V_{m n k}^{j} V_{p q r}^{i}+W_{m n k}^{j} W_{p q r}^{i}\right)\right]=\delta_{i 1} W_{p q r}^{i} P_{z} \equiv W_{p q r}^{1} P_{z} .
\end{aligned}
$$

Integration of (9) over the surface of the plate and application of the orthogonality relation (1) results in

$$
\zeta_{m n k}(t)+\omega_{m n k}^{2} \zeta_{m n k}(t)=\left(1 / N_{m n k}\right) \cdot Q_{m n k}(t),
$$

where the generalized forces $Q_{m n k}$ are given by:

$$
Q_{m n k}(t)=\int_{0}^{b} \int_{0}^{a} P_{z} \cdot W_{m n k}^{1} \mathrm{~d} x \mathrm{~d} y .
$$

With the initial conditions being homogeneous, the formal solution of (10a) can be presented as

$$
\zeta_{m n k}(t)=\left(1 / \omega_{m n k} N_{m n k}\right) \int_{0}^{t} Q_{m n k}(t) \cdot \sin \omega_{m n k}(t-\tau) \mathrm{d} \tau .
$$

Similarly, the primary response quantities of the plate within the equivalent singlelayer theories are obtained from

$$
\begin{aligned}
u(x, y, t) & =\sum_{m=1}^{\infty} \sum_{n=1}^{\infty} \sum_{k=1}^{K} U_{m n k}(x, y) \cdot \zeta_{m n k}(t), \\
v(x, y, t) & =\sum_{m=1}^{\infty} \sum_{n=1}^{\infty} \sum_{k=1}^{K} V_{m n k}(x, y) \cdot \zeta_{m n k}(t), \\
\psi_{x}(x, y, t) & =\sum_{m=1}^{\infty} \sum_{n=1}^{\infty} \sum_{k=1}^{5} \Psi_{x_{m n k}}(x, y) \cdot \zeta_{m n k}(t), \\
\psi_{y}(x, y, t) & =\sum_{m=1}^{\infty} \sum_{n=1}^{\infty} \sum_{k=1}^{5} \Psi_{y_{m n k}}(x, y) \cdot \zeta_{m n k}(t), \\
w(x, y, t) & =\sum_{m=1}^{\infty} \sum_{n=1}^{\infty} \sum_{k=1}^{K} W_{m n k}(x, y) \cdot \zeta_{m n k}(t),
\end{aligned}
$$

where $\zeta_{m n k}(t)$ are given by (11), with appropriate $N_{m n k}$ in various theories, and $Q_{m n k}(t)$ are defined as

$$
Q_{m n k}(t)=\int_{0}^{b} \int_{0}^{a} P_{z} \cdot W_{m n k} \mathrm{~d} x \mathrm{~d} y .
$$

In (12), $K=5$ in TSDPT and FSDPT and $K=3$ in CLPT. 
Note that the assumption of transverse inextensibility is made in all the equivalent single-layer theories considered here. For this reason the local nature of the impact damage cannot be taken into account in such theories. On the other hand, comparing the expression of the generalized forces $Q_{m n k}(t)$ in LWPT, $(10 \mathrm{~b})$, with that in the equivalent single-layer theories (13), clearly reveals the appropriateness of LWPT for the impact problems.

We express $N_{m n k}$ appearing in (4) as

$$
N_{m n k}=C_{m n k}^{1} \cdot C_{m n k}^{1} \cdot \hat{N}_{m n k},
$$

where

$$
\hat{N}_{m n k}=(a b / 4) I^{i j}\left(\frac{A_{m n k}^{i}}{C_{m n k}^{1}} \frac{A_{m n k}^{j}}{C_{m n k}^{1}}+\frac{B_{m n k}^{i}}{C_{m n k}^{1}} \frac{B_{m n k}^{j}}{C_{m n k}^{1}}+\frac{C_{m n k}^{i}}{C_{m n k}^{1}} \frac{C_{m n k}^{j}}{C_{m n k}^{1}}\right)
$$

Also the eigenfunctions can be presented as

$$
\begin{aligned}
U_{m n k}^{j} & =A_{m n k}^{j} \hat{U}_{m n}, \\
V_{m n k}^{j} & =B_{m n k}^{j} \hat{V}_{m n}, \\
W_{m n k}^{j} & =C_{m n k}^{j} \hat{W}_{m n}, j=1,2, \ldots, N+1,
\end{aligned}
$$

where $\hat{U}_{m n}=\cos \alpha_{m} x \sin \beta_{n} y, \hat{V}_{m n}=\sin \alpha_{m} x \cos \beta_{y} y, \hat{W}_{m n}=\sin \alpha_{m} x \sin \beta_{n} y$; and $\alpha_{m}$ and $\beta_{n}$ are defined in Nosier et al (1992).

With the help of (15), we can express the generalized forces $Q_{m n k}(t)$ as

$$
Q_{m n k}(t)=C_{m n k}^{1} \hat{Q}_{m n}(t),
$$

where $\hat{Q}_{m n}(t)=\int_{0}^{b} \int_{0}^{a} P_{z} \hat{W}_{m n}(x, y) \mathrm{d} x \mathrm{~d} y$.

Introducing (14) and (16) into (11) results in

$$
\zeta_{m n k}(t)=\frac{1}{\omega_{m n k}} \frac{1}{C_{m n k}^{1}} \frac{1}{\hat{N}_{m n k}} \cdot \hat{\zeta}_{m n k}(t)
$$

where

$$
\hat{\zeta}_{m n k}(t)=\int_{0}^{t} \hat{Q}_{m n}(t) \cdot \sin \omega_{m n k}(t-\tau) \mathrm{d} \tau
$$

Finally, upon substitution of (15) and (17) into (7) we obtain

$$
\begin{aligned}
& u^{j}(x, y, t)=\sum_{m=1}^{\infty} \sum_{n=1}^{\infty} \sum_{k=1}^{3(N+1)} \frac{1}{\omega_{m n k} \hat{N}_{m n k}} \cdot\left(\frac{A_{m n k}^{j}}{C_{m n k}^{1}}\right) \cdot U_{m n}(x, y) \cdot \hat{\zeta}_{m n k}(t), \\
& v^{j}(x, y, t)=\sum_{m=1}^{\infty} \sum_{n=1}^{\infty} \sum_{k=1}^{3(N+1)} \frac{1}{\omega_{m n k} \hat{N}_{m n k}} \cdot\left(\frac{B_{m n k}^{j}}{C_{m n k}^{1}}\right) \cdot V_{m n}(x, y) \cdot \hat{\zeta}_{m n k}(t), \\
& w^{j}(x, y, t)=\sum_{m=1}^{\infty} \sum_{n=1}^{\infty} \sum_{k=1}^{3(N+1)} \frac{1}{\omega_{m n k} \hat{N}_{m n k}} \cdot\left(\frac{C_{m n k}^{j}}{C_{m n k}^{1}}\right) \cdot W_{m n}(x, y) \cdot \hat{\zeta}_{m n k}(t) .
\end{aligned}
$$

Equations (19), in conjunction with (14), (15), (16), and (18), constitute the complete solution of the forced-vibration problem of a plate within LWPT.

Similarly, following the same line of reasoning, the primary response quantities of the plate according to various equivalent single-layer theories, as given by (12), can be obtained (see Nosier et al 1992). 


\subsection{Representation of impact load}

Determination of impact induced surface pressure and its spatial distribution is a major task in impact analysis. Often, the functional dependency of the pressure on temporal and spatial coordinates is assumed to be separable as

$$
P_{z}(x, y, t)=F(t) \cdot D(x, y),
$$

where $F(t)$ denotes the time-dependent amplitude of the pressure and the function $D(x, y)$ describes the spatial distribution of the impact pressure in contact area. The amplitude $F(t)$ is related to the identation of the plate at the contact point by a contact law.

Here we describe several models for presenting the spatial distribution of the pressure $P_{z}$ and evaluate the generalized forces $\hat{Q}_{m n}(t)$ in each model. Later, it will be demonstrated that the time history of the plate deflection is virtually independent of $D(x, y)$ as long as the total impulse, transmitted to the plate by the impactor, remains the same. The stress field in the contact region and in the vicinity of the contact area, is, however, very sensitive to the particular choice of pressure distribution.

Case-I - loading model: Assuming that the impact load can be described by a concentrated load at the point of contact, we have

$$
P_{z}(x, y, t)=F(t) \cdot \delta\left(x-a_{0}, y-b_{0}\right)
$$

where the generalized function $\delta$ is the two-dimensional Dirac's delta function. The generalized force $\hat{Q}_{m n}(t)$ is determined by substituting (15) and (21) into (16):

$$
\hat{Q}_{m n}(t)=F(t) \cdot K_{m n} \cdot H_{m n},
$$

where $K_{m n}=\sin \alpha_{m} \alpha_{0} \cdot \sin \beta_{n} b_{0}$, and $H_{m n}=1$.

Modelling the impact load as a concentrated load will result in an infinite shear force at the contact point in the plate. In cases II through V, it is assumed that the load is distributed over a known small rectangular or circular area. Further, its distribution will be uniform (cases II and IV), sinusoidal (case III), or spherical (case V).

Case-II-loading model: Assume that the impact load is uniformly distributed over a small rectangular area $(\bar{a} \times \bar{b})$ as shown in figure 1 . That is,

$$
P_{z}(x, y, t)= \begin{cases}\frac{F(t)}{\bar{a} \bar{b}}, & a_{0}-\frac{\bar{a}}{2} \leqslant x \leqslant a_{0}+\frac{\bar{a}}{2}, b_{0}-\frac{\bar{b}}{2} \leqslant y \leqslant b_{0}+\frac{\bar{b}}{2}, \\ 0, & \text { otherwise. }\end{cases}
$$

Evaluating $\hat{Q}_{m n}(t)$ and expressing it as in (22), we have

$$
H_{m n}=\left(\sin \alpha_{m} \frac{\bar{a}}{2} / \alpha_{m} \frac{\bar{a}}{2}\right)\left(\sin \beta_{n} \frac{\bar{b}}{2} / \beta_{n} \frac{\bar{b}}{2}\right) .
$$

In cases $\mathrm{I}$ through $\mathrm{V}, K_{m n}$ will be as given earlier.

Case-III-loading model: Here we assume that a cosine-shaped load is distributed 


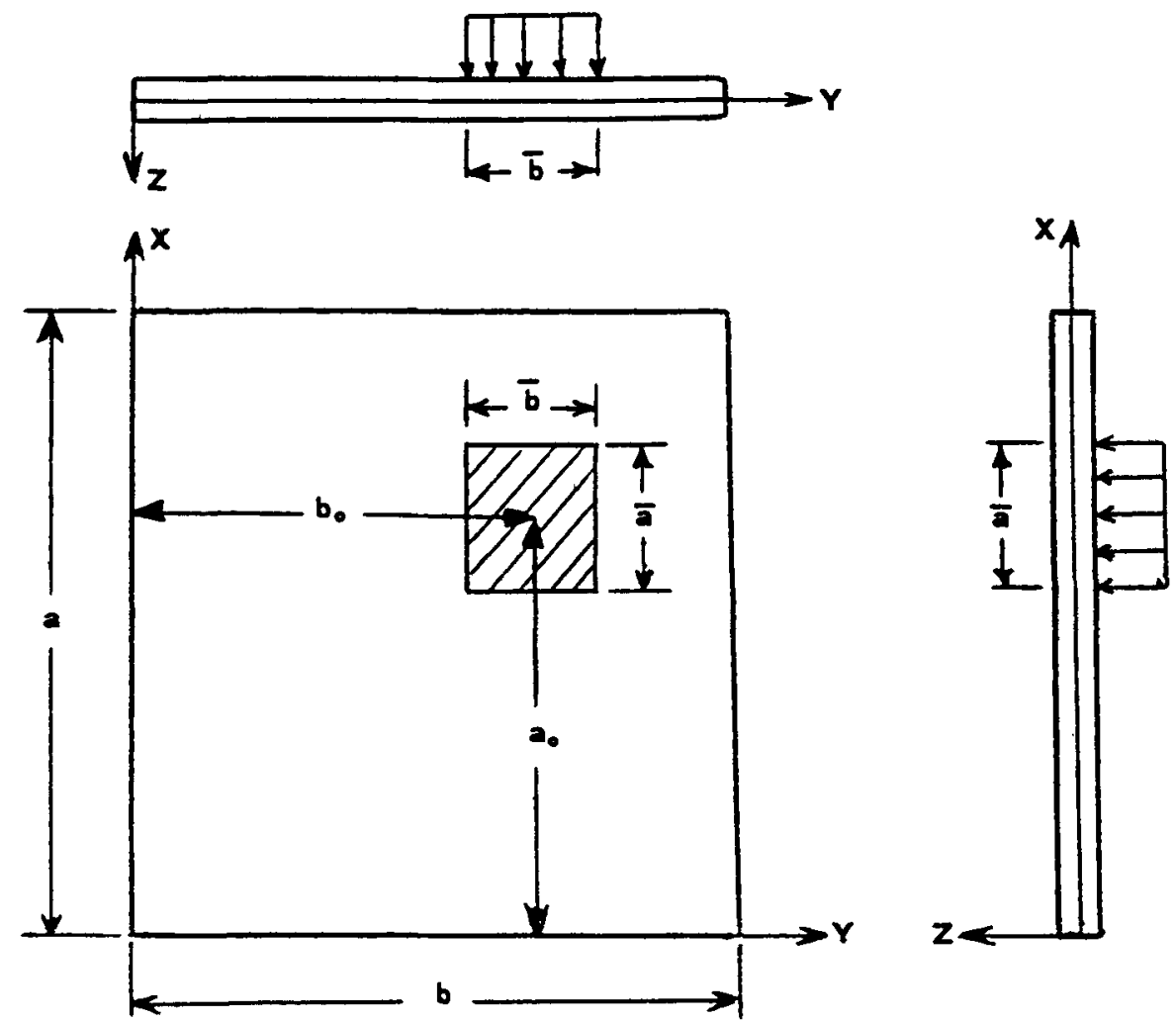

Figure 1. The impact load modelled as a uniform load distributed over a small rectangular region $(\bar{a} \times \bar{b})$; case-II loading model.

over a small rectangular area. Hence

$$
P_{z}(x, y, t)= \begin{cases}F(t) \cdot \frac{\pi^{2}}{4 \bar{a} \bar{b}} \cdot \cos \frac{\pi}{\bar{a}}\left(x-a_{0}\right) \cdot \cos \frac{\pi}{\bar{b}}(y-\bar{y}), & a_{0}-\frac{\bar{a}}{2} \leqslant x \leqslant a_{0}+\frac{\bar{a}}{2} \\ 0, & b_{0}-\frac{\bar{b}}{2} \leqslant y \leqslant b_{0}+\frac{\bar{b}}{2}, \\ \text { otherwise, }\end{cases}
$$

and

$$
H_{m n}=\left\{\cos \alpha_{m} \frac{\bar{a}}{2} /\left[1-\left(\alpha_{m} \frac{\bar{a}}{\pi}\right)^{2}\right]\right\} \cdot\left\{\cos \beta_{n} \frac{\bar{b}}{2} /\left[1-\left(\beta_{n} \frac{\bar{b}}{\pi}\right)^{2}\right]\right\} .
$$

Case-IV-loading model: It is more realistic to model the contact area as a circular region, at least for an isotropic plate. Also, in the case of orthotropic plates the contact area is only slightly elliptical (see Greszczuk 1982). As a first approximation we assume that the impact load is uniformly distributed over this circular contact area, see figure 2 . That is,

$$
P_{z}(x, y, t)= \begin{cases}F(t) / \pi R_{0}^{2}, & \text { over the shaded area, } \\ 0, & \text { otherwise }\end{cases}
$$



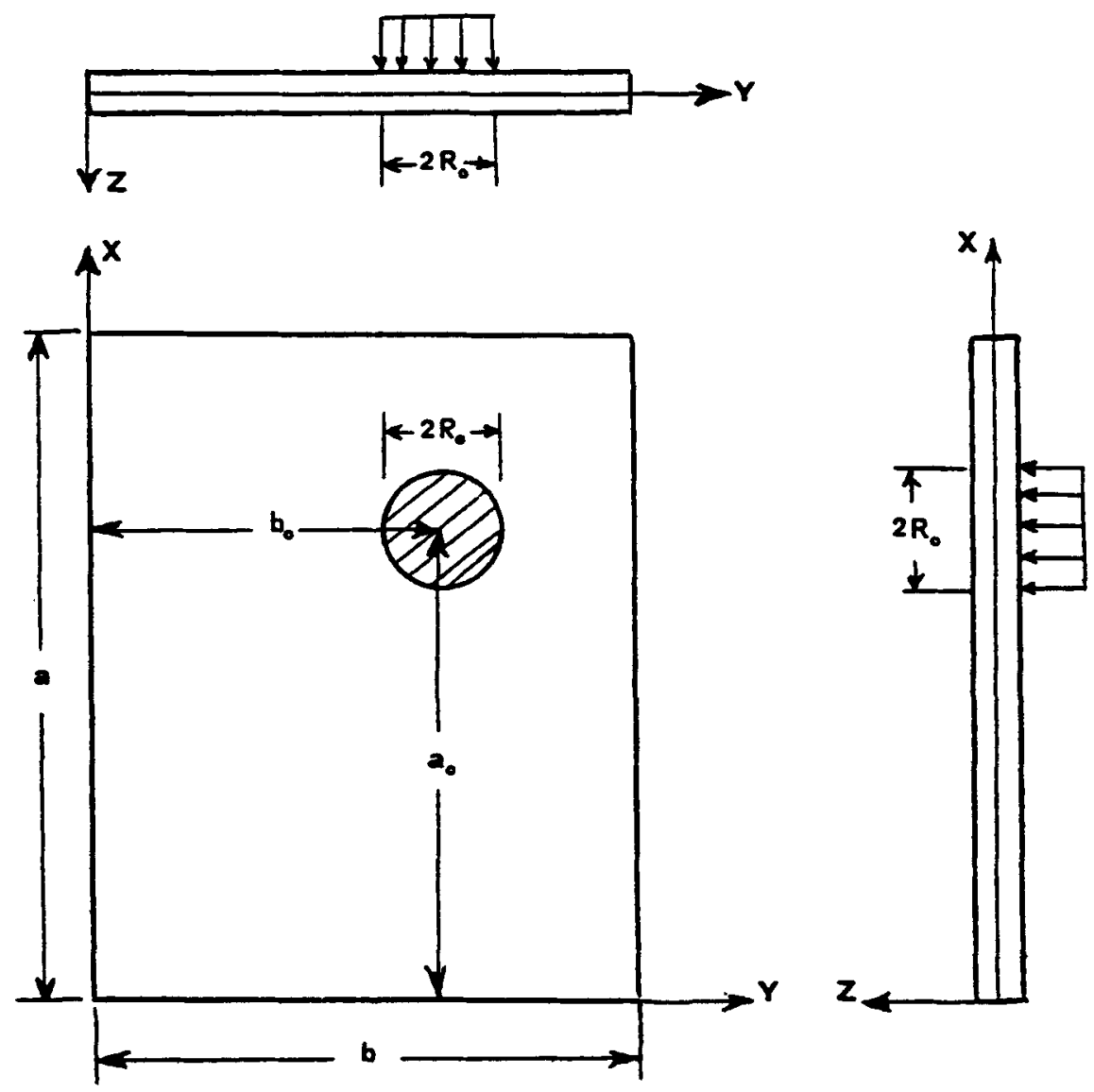

Figure 2. The impact load modelled as a uniform load distributed over a small circular region; case-IV loading model.

and, therefore, (see appendix D in Nosier et al 1992)

$$
H_{m n}=2\left(J_{1}(\bar{R})\right) / \bar{R},
$$

where $\bar{R}=\left(\alpha_{m}^{2}+\beta_{n}^{2}\right)^{1 / 2} R_{o} ;$ and $J_{1}$ is the Bessel function of the first kind and of order one.

Case-V-loading model. In the Hertzian contact law, the distribution of the contact load in the circular contact area is found to be spherically shaped (Timoshenko \& Goodier 1970). It is to be remembered, however, that in the Hertz model both the target and the projectile were assumed to be isotropic and elastic; the target was assumed semi-infinite. For a spherically-shaped load we have (see figure 3):

$$
P_{z}(x, y, t)= \begin{cases}F(t) \frac{3}{2 \pi R_{0}^{2}}\left[1-\frac{1}{R_{0}^{2}}\left(\bar{x}^{2}+\bar{y}^{2}\right)\right]^{1 / 2}, & \text { in the shaded area } \\ 0, & \text { otherwise }\end{cases}
$$



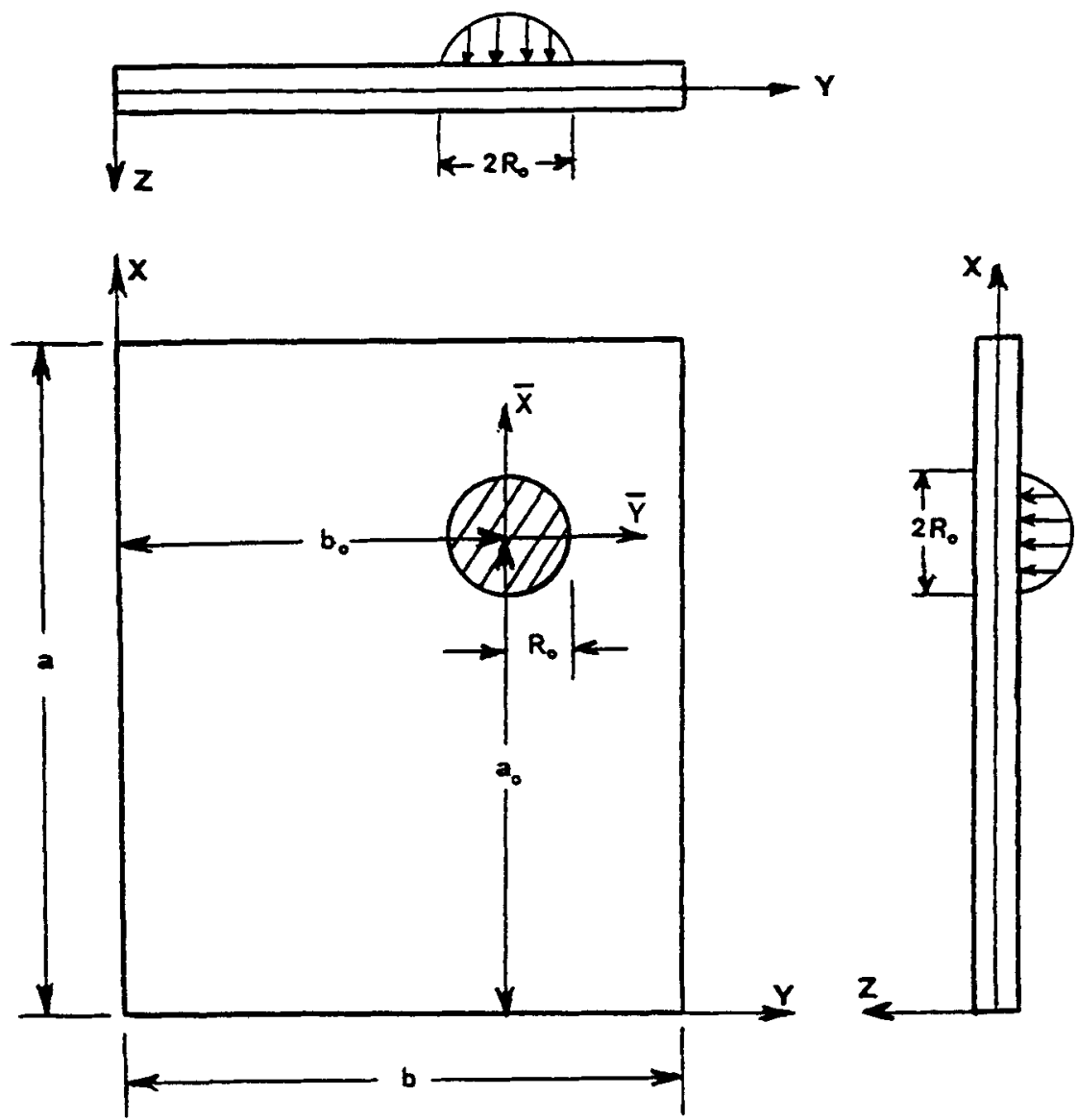

Figure 3. The impact load modelled as a spherically-shaped load over a small circular region; case-V loading model.

In this case we have (see appendix D in Nosier et al 1992):

$$
H_{m n}=\left(3 / \bar{R}^{2}\right)[(\sin \bar{R}) / \bar{R}-\cos \bar{R}]
$$

where $\bar{R}$ is as defined earlier in conjunction with (28).

Having expressed the generalized forces in terms of the unknown amplitude of the impact load, the displacement of the plate at the contact point $\left(x=a_{o}, y=b_{o}\right)$ according to, for example, LWPT, is obtained by substituting (22) into (18) and the result so obtained into (19), with $j=1$ :

$$
\begin{aligned}
u_{3}\left(a_{o}, b_{o},-h / 2, t\right)= & w^{1}\left(a_{o}, b_{o}, t\right)= \\
& \sum_{m=1}^{\infty} \sum_{n=1}^{\infty} \sum_{k=1}^{3(N+1)} \bar{B}_{m n k} \int_{0}^{t} F(\tau) \sin \omega_{m n k}(t-\tau) \mathrm{d} \tau,
\end{aligned}
$$

where $\bar{B}_{m n k}=\left(1 / \omega_{m n k} N_{m n k}\right) K_{m n}^{2} H_{m n}$. Similarly, the plate displacement at the contact point according to HSDPT, FSDPT, and CLPT can be obtained (see Nosier et al 1992).

By invoking a contact law, the displacement and the amplitude of the impact force along with other pertinent response quantities can be determined. 
Case-VI - loading model - special treatment: In cases II through V we have assumed that the impact pressure is distributed in a certain fashion over a known contact region. Generally, however, this contact area is unknown and changes with time. On the other hand, we mentioned that in the Hertz model, being based on completely elastic behaviour, the load distribution is spherically shaped. Furthermore, in Hertz's law of contact the magnitude of the total impact load $F(t)$ is related to the indentation $\alpha(t)$, at the contact point, as

$$
F(t)=K_{2} \alpha^{3 / 2}(t)
$$

with $K_{2}$ being a constant coefficient.

We can relate the radius $R_{o}$ of the circular contact area in the case- $V$ loading to the force $F(t)$ by using the relationship

$$
R_{o}(t)=R_{S}^{1 / 2} \alpha^{1 / 2}(t),
$$

which is valid for elastic deformation (Hertzian) law. That is, elimination of $\alpha(t)$ from (33), with the help of (32), results in

$$
R_{o}(t)=\left(R_{S}^{1 / 2} / K_{2}^{1 / 3}\right) \cdot F^{1 / 3}(t),
$$

where $R_{S}$ denotes the radius of the spherical impactor.

Upon substitution of (34) into (30) and the result so obtained along with (22) into (18), we obtain

$$
\begin{array}{r}
\hat{\zeta}_{m n k}(t)=\left(3 k_{m n} / \gamma_{m n}^{3}\right) \int_{0}^{t}\left\{\sin \left[\gamma_{m n} F^{1 / 3}(\tau)\right]-\gamma_{m n} F^{1 / 3}(\tau) \cdot \cos \left[\gamma_{m n} F^{1 / 3}(\tau)\right]\right\} \\
\times \sin \omega_{m n k}(t-\tau) \mathrm{d} \tau
\end{array}
$$

where $\gamma_{m n}=\left(\alpha_{m}^{2}+\beta_{n}^{2}\right)^{1 / 2}\left(R_{S}^{1 / 2} / K_{2}^{1 / 3}\right)$. The displacement of the plate at the contact point is found by substituting (35) in (19), with $j=1$ :

$$
\begin{aligned}
& u_{3}\left(a_{o}, b_{o},-h / 2, t\right)=w^{1}\left(a_{o}, b_{o}, t\right) \\
& =\sum_{m=1}^{\infty} \sum_{n=1}^{\infty} \sum_{k=1}^{3(N+1)} \bar{B}_{m n k} \int_{0}^{t}\left\{\sin \left[\gamma_{m n} F^{1 / 3}(\tau)\right]-\gamma_{m n} F^{1 / 3}(\tau) \cdot \cos \left[\gamma_{m n} F^{1 / 3}(\tau)\right]\right\} \\
& \times \sin \omega_{n m k}(t-\tau) \mathrm{d} \tau .
\end{aligned}
$$

Similarly, the displacement at the contact point according to the equivalent single-layer theories is given by:

$$
\begin{array}{r}
u_{3}\left(a_{o}, b_{o},-h / 2, t\right)=w\left(a_{o}, b_{o}, t\right) \\
=\sum_{m=1}^{\infty} \sum_{n=1}^{\infty} \sum_{k=1}^{K} \bar{B}_{m n k} \int_{0}^{t}\left\{\sin \left[\gamma_{m n} F^{1 / 3}(\tau)\right]-\gamma_{m n} F^{1 / 3}(\tau) \cdot \cos \left[\gamma_{m n} F^{1 / 3}(\tau)\right]\right\} \\
\times \sin \omega_{m n k}(t-\tau) \mathrm{d} \tau,
\end{array}
$$

where $\bar{B}_{m n k}=3 K_{m n}^{2} /\left(\gamma_{m n}^{3} \omega_{m n k} \hat{N}_{m n k}\right)$ in all theories, where the appropriate expressions for $\hat{N}_{m n k}$ must be used.

In the case-VI model, the contact area, being time-dependent, is increased in the loading process from zero to a maximum when the force amplitude is maximum and 
then decreases in the unloading process until it becomes zero again at the time of rebound.

In the next section, two algorithms will be introduced for the determination of the impact force and responses quantities for loading cases I through $\mathrm{V}$. The determination of impact force and response of the plate for the case-VI loading model will be followed in a separate section. The variation of the total impact force $F(t)$ and the transverse displacement of the laminate will, of course, be approximately identical for all the loading models we are considering here. This conclusion is also valid as far as the stress field away from the contact point is concerned. The significance of the various models will become apparent only when we consider the stress field in the vicinity of the impact zone.

\section{Impact response. Analysis}

The direct measurement of the dynamic contact force is a complicated task due to the existence of a wide range of plate and impactor parameters. It will, therefore, be assumed that the vibrations of the impactor and the laminate can be neglected so that an elastostatic indentation law can be used. That is, the impact duration will be assumed to be long compared to the stress-wave transit times in the impactor and the laminate.

\subsection{Contact laws}

The most famous elastic contact law was derived by Hertz (see (32)) for the contact of two spheres of elastic isotropic materials. The contact between a sphere on a halfspace is a limiting case. In (32) $\alpha$ denotes the indentation and the contact coefficient $K_{2}$ is given by

$$
K_{2}=(4 / 3)\left[R_{s}^{1 / 2} /\left(\delta_{1}+\delta_{2}\right)\right]
$$

where $\delta_{1}=\left(1-v_{s}^{2}\right) / E_{s}$; and $\delta_{2}=\left(1-v^{2}\right) / E$. In (38), $R_{s}$ denotes the radius of the spherical impactor. Also $\left(v_{s}, E_{s}\right)$ and $(v, E)$ are the Poisson ratio and Young's modulus of the impactor and the half-space, respectively. The indentation law for an isotropic spherical impactor and a transversely isotropic half-space (see Conwag 1956) is also given by (32). The $x-y$ plane is assumed to be the plane of isotropy. The expressions of $K_{2}$ and $\delta_{1}$ are again given as mentioned earlier in conjunction with (38), and

where

$$
\begin{aligned}
\delta_{2}=\bar{A}_{22}^{1 / 2}\left\{\left[\left(\bar{A}_{11} \bar{A}_{22}\right)^{1 / 2}+G_{z}\right]^{2}-\left(\bar{A}_{12}+G_{z}\right)^{2}\right\}^{1 / 2} \\
\div\left\{2 G_{z}^{1 / 2}\left(\bar{A}_{11} \bar{A}_{22}-\bar{A}_{22}^{2}\right)\right\}
\end{aligned}
$$

$$
\begin{array}{r}
\bar{A}_{11}=E_{z} /\left\{1-\left[2 v_{z}^{2} E_{z} /(1-v) E\right]\right\}, \quad A_{22}=\left[\left(E / E_{z}\right)-v_{2}^{2}\right] \bar{A}_{11} /\left(1-v^{2}\right), \\
\bar{A}_{12}=v_{z} \bar{A}_{11} /(1-v) .
\end{array}
$$

Here, $v$ and $E$ are the Poisson ratio and Young's modulus in the plane of isotropy ( $x-y$ plane), respectively. That is, $v=v_{12}=v_{21}$ and $E=E_{1}=E_{2}$. Also, $v_{2}=v_{13}=v_{23}$ and $E_{z}=E_{3}$ with the shear modulus $G_{z}=G_{13}=G_{23}$.

When the plate is laminated of orthotropic layers, Sun (1977) employed the Hertz law with $\delta_{2}=1 / E_{2}$ where $E_{2}$ is the Young modulus transverse to the fibre direction 
in the $x-y$ plane. The value of $K_{2}$ so determined was observed by Yang \& Sun (1981) to be higher than that obtained experimentally for a cross-ply laminate. At the present time the exact value of $\delta_{2}$ for an orthotropic half-space is not known. However, the approximate numerical solution for $\delta_{2}$ shows that $\delta_{2}$ is relatively insensitive to the in-plane fibre orientation (Greszczuk 1975). For this reason, it is concluded by Greszczuk (1982) that for an orthotropic target, (39) can be used, as a first approximation, if average in-plane properties, say, $v=\left(v_{12}+v_{21}\right) / 2, E=\left(E_{1}+E_{2}\right) / 2$ are used. It is, however, noted by Greszczuk (1975) that the properties that influence $\delta_{2}$ the most are those associated with the thickness $(z-)$ direction. When an isotropic sphere is pressed into either an isotropic or transversely isotropic half-space, the area of contact is circular according to elasticity solutions. Furthermore, the radius $R_{0}$ of this area is related to the radius $R_{s}$ of the sphere and the indentation $\alpha$ according to (see Timoshenko \& Goodier 1970): $R_{0}=R_{s}^{1 / 2} \alpha^{1 / 2}$.

The Hertzian contact law, being an elastic law, does not account for permanent indentation. Permanent indentation may often take place even at relatively low loading levels in composite targets and, therefore, the unloading curve may in general be different from the loading curve. The experimental studies of Tan \& Sun (1982) indicated that the following approximate relations can be used as a proper contact law for composite targets:

where

$$
\begin{array}{ll}
\text { loading: } & F=K_{2} \alpha^{3 / 2}, \\
\text { unloading: } & F=F_{m}\left[\left(\alpha-\alpha_{o}\right) /\left(\alpha_{m}-\alpha_{o}\right)\right]^{q}, \\
\text { reloading: } & F=F_{m}\left[\left(\alpha-\alpha_{0}\right)\left(\alpha_{m}-\alpha_{o}\right)\right]^{3 / 2},
\end{array}
$$

$$
\alpha_{o}=\left\{\begin{array}{lll}
\beta\left(\alpha_{m}-\alpha_{p}\right), & \text { if } \alpha_{m}>\alpha_{p}, \\
0, & \text { if } \quad \alpha_{m}<\alpha_{p} .
\end{array}\right.
$$

In (40), $F_{m}$ denotes the maximum force reached before unloading, $\alpha_{m}$ is the corresponding indentation, and $\alpha_{0}$ is the permanent indentation. The values of $K_{2}, \beta, q$, and $\alpha_{p}$ are found experimentally. As a first approximation, however, $K_{2}$ can be determined from (38), with $\delta_{2}=1 / E_{2}$.

\subsection{Determination of impact force}

In this section we are concerned with the determination of the impact force when the transverse load is represented as any one of the cases I through V loading models. In determining the impact force we follow the basic approach developed by Timoshenko (1913), who studied the impact of an isotropic beam by a sphere (also see Goldsmith 1960). Denoting by $m_{0}, v_{0}$, and $z$ the mass of the impactor, the velocity of the impactor at the moment of impact, and the displacement of the impactor after the impact, we have

$$
z(t)=v_{0} t-\left(1 / m_{0}\right) \int_{0}^{t} F(\tau)(t-\tau) \mathrm{d} \tau .
$$

Furthermore, if impact on the laminate occurs at $\left(x=a_{0}, y=b_{0}\right)$, we have (see figure 4):

$$
\alpha(t)=z(t)-u_{3}\left(a_{0}, b_{0},-h / 2, t\right)
$$




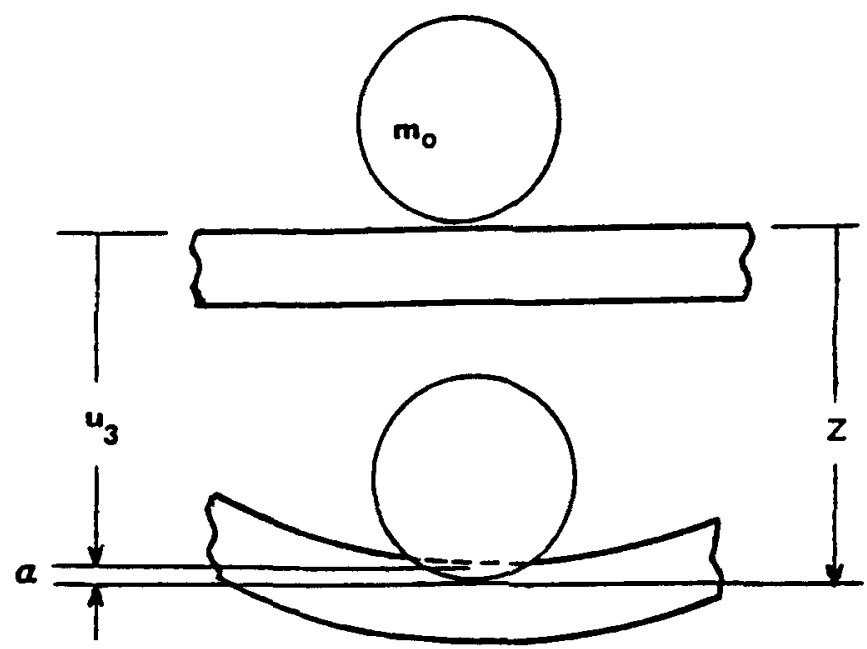

Figure 4. A plate before and after impact.

where $u_{3}\left(a_{0}, b_{0},-h / 2, t\right)$ is the displacement of the laminate, at the point of impact, whose expression is given earlier. Using Hertz contact law (see Nosier et al 1992), we obtain

$$
\begin{aligned}
K_{2}^{-2 / 3} F^{2 / 3}(t)=v_{0} t-\left(1 / m_{0}\right) & \int_{0}^{t} F(\tau)(t-\tau) \mathrm{d} \tau \\
& -\sum_{m=1}^{\infty} \sum_{n=1}^{\infty} \sum_{k=1}^{K} \bar{B}_{m n k} \int_{0}^{t} F(\tau) \cdot \sin \omega_{m n k}(t-\tau) \mathrm{d} \tau,
\end{aligned}
$$

where $\bar{B}_{m n k}$ and $K$ have been defined earlier. Upon solving (43) the time history of the impact force will be obtained. Here we use two different numerical schemes to determine the approximate values of the impact force at various time steps. Our first approach will be to assume a linear variation for the force during each small time increment. This approach will be very similar to the one presented by Sankar \& Sun (1985a) but it will have one essential difference. Sankar \& Sun (1985a) have taken the force variation as

$$
F(t)=\sum_{i=0,1,2 \ldots} q_{i} R\langle t-i \Delta t\rangle
$$

where the $q_{i}$ 's are unknowns that decide the contact force history, and $R\langle t-i \Delta t\rangle$ is the function defined as follows:

$$
R\left\langle t-t_{0}\right\rangle= \begin{cases}0, & \text { for } t \leqslant t_{0} \\ \left(t-t_{0}\right) / \Delta t, & \text { for } t_{0} \leqslant t \leqslant t_{0}+\Delta t, \\ 1, & \text { for } t \geqslant t_{0}+\Delta t\end{cases}
$$

In our first approach we alternatively assume that

$$
F(t)=\sum_{i=1,2, \ldots} f_{i} \Phi_{i}(t)
$$

where $\Phi_{i}$ 's are the linear Lagrange form interpolation polynomials (the so-called hat 
functions). That is

$$
\Phi_{i}(t)= \begin{cases}0, & \text { for } t \leqslant(i-1) \Delta t, \\ \psi_{i}^{1}=(1 / \Delta t)[t(i-1) \Delta t], & \text { for } \quad(i-1) \Delta t \leqslant t \leqslant i \Delta t, \\ \psi_{i}^{2}=(1 / \Delta t)[(i-1) \Delta t-t], & \text { for } i \Delta t \leqslant t \leqslant(i+1) \Delta t, \\ 0, & \text { for } t \geqslant(i-1) \Delta t .\end{cases}
$$

From (46) and (47) at time $t=i \Delta t$ we have $F(i \Delta t)=f_{i}$. Hence, $f_{1}, f_{2}, \ldots$ are the magnitudes of the total impact force at times equal to $\Delta t, 2 \Delta t, \ldots$, respectively. Also when $(i-1) \Delta t \leqslant t \leqslant i \Delta t$, we have

$$
F(t)=\left[i f_{i-1}-(i-1) f_{i}\right]+(1 / \Delta t)\left(f_{i}-f_{i-1}\right) t, \quad i=1,2, \ldots,
$$

with $f_{0}=F(0)=0$. Next we assume that $t=p \Delta t(p=1,2, \ldots)$ and evaluate the two integrals appearing in (43), with the help of (48), and obtain

and

$$
\int_{0}^{t} F(\tau)(t-\tau) d \tau=\left[(\Delta t)^{2} / 6\right] \cdot f_{p}+\delta_{p} \sum_{i=1}^{p-1}(p-i) \cdot(\Delta t)^{2} \cdot f_{i}
$$

$$
\begin{aligned}
& \int_{0}^{i} F(\tau) \cdot \sin \omega_{m n k}(t-\tau) \mathrm{d} \tau=\left(\frac{1}{\omega_{m n k}}-\frac{1}{\Delta t \omega_{m n k}^{2}} \sin \omega_{m n k} \Delta t\right) \cdot f_{p} \\
&+\delta_{p} \sum_{i=1}^{p-1}\left[\frac{2}{\Delta t \omega_{m n k}^{2}}\left(1-\cos \omega_{m n k} \Delta t\right) \cdot \sin \omega_{m n k}(p-i) \Delta t\right] \cdot f_{i}
\end{aligned}
$$

where $\delta_{p}=0$ for $p=1$ and $\delta_{p}=1$ for all other values of $p$. Also at time $t=p \Delta t$ let $F(p \Delta t)=f_{p}$. Then from (43) and (49), we have:

$$
a_{p} f_{p}^{2 / 3}+b_{p} f_{p}+c_{p}=0
$$

where the constant coefficients $a_{p}, b_{p}$, and $c_{p}$ are given in Nosier et al (1992). Now by introducing the new variable $g_{p}$ as $f_{p}=g_{p}^{3}$ in (50) we obtain

$$
b_{p} g_{p}^{3}+a_{p} g_{p}^{2}+c_{p}=0
$$

whose roots can be found exactly, and the magnitudes of the total impact force $F(t)$ at various times $p \Delta t(p=1,2, \ldots)$ can be obtained.

When the experimental contact law of Tan \& Sun (1982) (40) is employed, (50) will be valid for the loading process. However, using the expression for $\alpha(t)$ from (40b) in (43) and carrying out the above analysis we found that for the unloading process the magnitudes of the total impact force at various times $t=p \Delta t$ are found from

$$
\bar{K}_{3} f_{p}^{1 / q}+b_{p} f_{p}+c_{p}+\alpha_{o}=0
$$

where $\bar{K}_{3}, b_{p}$ and $c_{p}$ are given by Nosier et al (1992). Similarly, the magnitudes of the impact force at various times are obtained from

$$
\bar{K}_{4} f_{p}^{2 / 3}+b_{p} f_{p}+c_{p} \alpha_{0}=0,
$$

where $\bar{K}_{4}$ is given by Nosier et al (1992). It is to be noted that when $\alpha_{0}=0,(32 b)$ becomes identical to $(50)$. That is, the reloading contact law of Tan \& Sun (1982) 
will be identical to the Hertzian contact law. The manner in which (52a) will be solved depends on the experimentally evaluated value of $q$. The experimental results of Tan \& Sun (1982) indicated that this value varied between 2 and 3. For $q=2 \cdot 5$, (52a) will be solved numerically. However for $q=2$ and $q=3$ (52a) can be transformed, respectively, into a quadratic equation and a cubic equation which can be solved exactly.

The impactor position at any time $t=p \Delta t$ can be obtained as:

$$
z(p \Delta t)=v_{o} \cdot(p \Delta t)-\frac{1}{6} \frac{(\Delta t)^{2}}{m_{0}} \cdot f_{p}-\frac{\delta_{p}}{m_{0}} \sum_{i=1}^{p-1}(p-i) \cdot(\Delta t)^{2} \cdot f_{i} .
$$

The transverse displacement of the laminate at the point of contact according to the layerwise theory and the equivalent single-layer theories can be written as

$$
\begin{array}{r}
u_{3}\left(a_{0}, b_{0},-h / 2, p \Delta t\right)=\left[\sum_{m=1}^{\infty} \sum_{n=1}^{\infty} \sum_{k=1}^{K} \bar{B}_{m n k} \cdot\left(\frac{1}{\omega_{m n k}}-\frac{1}{\Delta t \omega_{m n k}^{2}} \sin \omega_{m n k} \Delta t\right)\right] \cdot f_{p} \\
+\delta_{p} \sum_{i=1}^{p-1} \sum_{m=1}^{\infty} \sum_{n=1}^{\infty} \sum_{k=1}^{K} \bar{B}_{m n k}\left[\frac{2}{\Delta t \omega_{m n k}^{2}}\left(1-\cos \omega_{m n k} \Delta t\right) \cdot \sin \omega_{m n k}(p-i) \Delta t\right] \cdot f_{i},
\end{array}
$$

where the values of $K$ for various theories have been given earlier.

Next, in the second scheme, we assume that the contact force variation is cubic in time during each time increment and employ the global Hermite cubic interpolation polynomials.

$$
F(t)=\sum_{i=1,2, \ldots}\left[f_{i} \Phi_{i}(t)+\bar{f}_{i} \bar{\Phi}_{i}(t)\right]
$$

where $\Phi_{i}$ 's and $\Phi_{i}$ 's are given as:

and

$$
\Phi_{i}(t)= \begin{cases}0, & \text { for } t \leqslant(i-1) \Delta t \\ \psi_{i}^{1}(t), & \text { for } \quad(i-1) \Delta t \leqslant t \leqslant i \Delta t, \\ \psi_{i}^{2}(t), & \text { for } i \Delta t \leqslant t \leqslant(i+1) \Delta t, \\ 0, & \text { for } t \geqslant(i+1) \Delta t\end{cases}
$$

$$
\bar{\Phi}_{i}(t)= \begin{cases}0, & \text { for } t \leqslant(i-1) \Delta t \\ \bar{\psi}_{i}^{1}(t), & \text { for } \quad(i-1) \Delta t \leqslant t \leqslant i \Delta t \\ \bar{\psi}_{i}^{2}(t), & \text { for } \quad(i-1) \Delta t \leqslant t \leqslant(i+1) \Delta t \\ 0, & \text { for } t \geqslant(i+1) \Delta t\end{cases}
$$

where

$$
\begin{aligned}
& \psi_{i}^{1}(t)=\left(3 / \Delta t^{2}\right)[t-(i-1) \Delta t]^{2}-\left(2 / \Delta t^{3}\right)[t-(i-1) \Delta t]^{3}, \\
& \psi_{i}^{2}(t)=1-\left(3 / \Delta t^{2}\right)(t-i \Delta t)^{2}+\left(2 / \Delta t^{3}\right)(t-i \Delta t)^{3}, \\
& \bar{\psi}_{i}^{1}(t)=\left(1 / \Delta t^{2}\right)[t-(i-1) \Delta t]^{3}-(1 / \Delta t)[t-(i-1) \Delta t]^{2}, \\
& \bar{\psi}_{i}^{2}(t)=(t-i \Delta t)-(2 / \Delta t)(t-i \Delta t)^{2}+\left(1 / \Delta t^{2}\right)(t-i \Delta t)^{3} .
\end{aligned}
$$

In (56) we have

$$
f_{i}=F(i \Delta t) \text { and } \bar{f}_{i}=\left.\frac{\mathrm{d} F}{\mathrm{~d} t}\right|_{t=i \Delta t}
$$


That is, $f_{i}$ is, as before, the magnitude of the total impact force at time $t=i \Delta t$ and $\bar{f}_{i}$ is the slope of the force-time curve at time $t=i \Delta t$. Furthermore, we have $f_{0}=F(0)=0$ and $\bar{f}_{0}=\left.\frac{\mathrm{d} F}{\mathrm{~d} t}\right|_{t=0}=0$. The last result can be obtained by differentiation of (32) and noting that $\alpha(0)=0$. So, in summary, we are required to determine $f_{i}$ and $\bar{f}_{i}$ at each time step $(t=i \Delta t)$. For this purpose we need another equation besides (43). This second equation is obtained by direct differentiation of (43):

$$
\begin{aligned}
(2 / 3) K_{2}^{-2 / 3} \dot{F}(t) \cdot F^{-1 / 3}(t) & =v_{0}-\left(1 / m_{0}\right) \int_{0}^{t} F(\tau) \mathrm{d} \tau \\
& -\sum_{m=1}^{\infty} \sum_{n=1}^{\infty} \sum_{k=1}^{K} \omega_{m n k} \bar{B}_{m n k} \int_{0}^{t} F(\tau) \cdot \cos \omega_{m n k}(t-\tau) \mathrm{d} \tau .
\end{aligned}
$$

When $(i-1) \Delta t \leqslant t \leqslant i \Delta t$, we have

$$
F(t)=f_{i-1} \psi_{i-1}^{2}+\bar{f}_{i-1} \bar{\psi}_{i-1}^{2}+f_{i} \psi_{i}^{1}+\bar{f}_{i} \bar{\psi}_{i}^{1}, \quad i=1,2, \ldots
$$

We evaluate the four integrals appearing in (43) and (58) at time $t=p \Delta t(p=1,2, \ldots)$ :

$$
\begin{aligned}
& \int_{0}^{t} F(\tau) \cdot(t-\tau) \mathrm{d} \tau=\int_{0}^{p \Delta t} F(\tau) \cdot(p \Delta t-\tau) \mathrm{d} \tau \\
& =\frac{3}{20}(\Delta t)^{2} \cdot f_{p}-\frac{1}{30}(\Delta t)^{3} \cdot \bar{f}_{p}+\delta_{p} \sum_{i=1}^{p-1}(\Delta t)^{2}\left[(p-i) \cdot f_{i}-\frac{\Delta t}{15} \cdot \bar{f}_{i}\right], \\
& \int_{0}^{t} F(\tau) \cdot \sin \omega_{m n k}(t-\tau) d \tau=\int_{0}^{p \Delta t} F(\tau) \cdot \sin \omega_{m n k}(p \Delta t-\tau) \mathrm{d} \tau \\
& =\left[\frac{1}{\omega_{m n k}}-\frac{12}{\Delta t^{3} \omega_{m n k}^{4}} \sin \omega_{m n k} \Delta t+\frac{6}{\Delta t^{2} \omega_{m n k}^{3}}\left(1+\cos \omega_{m n k} \Delta t\right)\right] \cdot f_{p} \\
& +\left[\frac{6}{\Delta t^{2} \omega_{m n k}^{4}}-\sin \omega_{m n k} \Delta t-\frac{2}{\Delta t \omega_{m n k}^{3}}\left(2+\cos \omega_{m n k} \Delta t\right)\right] \cdot \bar{f}_{p} \\
& +\delta_{p} \cdot \sum_{i=1}^{p-1}\left\{\sin \omega_{m n k} \Delta t(p-i)\left[\frac{24}{\Delta t^{3} \omega_{m n k}^{4}}\left(1-\cos \omega_{m n k} \Delta t\right)-\frac{12}{\Delta t^{2} \omega_{m n k}^{3}} \sin \omega_{m n k} \Delta t\right]\right\} \cdot f_{i} \\
& +\delta_{p} \cdot \sum_{i=1}^{p-1}\left\{\cos \omega_{m n k} \Delta t(p-i)\left[\frac{12}{\Delta t^{2} \omega_{m n k}^{4}} \sin \omega_{m n k} \Delta t-\frac{4}{\Delta t \omega_{m n k}^{3}}\left(2+\cos \omega_{m n k} \Delta t\right)\right]\right\} \cdot \bar{f}_{i}, \\
& \int_{0}^{t} F(\tau) \mathrm{d} \tau=\int_{0}^{p \Delta t} F(\tau) \mathrm{d} \tau=\frac{1}{2} \Delta t \cdot f_{p}-\frac{1}{12}(\Delta t)^{2} \cdot \bar{f}_{p}+\delta_{p} \cdot \sum_{i=1}^{p-1} \Delta t \cdot f_{i}, \\
& \int_{0}^{t} F(\tau) \cdot \cos \omega_{m n k}(t-\tau) \mathrm{d} \tau=\int_{0}^{p \Delta t} F(\tau) \cdot \cos \omega_{m n k}(p \Delta t-\tau) \mathrm{d} \tau \\
& =\left[\frac{12}{\Delta t^{3} \omega_{m n k}^{4}}\left(1-\cos \omega_{m n k} \Delta t\right)-\frac{6}{\Delta t^{2} \omega_{m n k}^{3}} \sin \omega_{m n k} \Delta t\right] \cdot f_{p}
\end{aligned}
$$




$$
\begin{aligned}
& +\left[\frac{1}{\omega_{m n k}^{2}}+\frac{2}{\Delta t \omega_{m n k}^{3}} \sin \omega_{m n k} \Delta t-\frac{6}{\Delta t^{2} \omega_{m n k}^{4}}\left(1-\cos \omega_{m n k} \Delta t\right)\right] \cdot \bar{f}_{p} \\
& +\delta_{p} \cdot \sum_{i=1}^{p-1}\left\{\cos \omega_{m n k} \Delta t(p-i)\left[\frac{24}{\Delta t^{3} \omega_{m n k}^{4}}\left(1-\cos \omega_{m n k} \Delta t\right)-\frac{12}{\Delta t^{2} \omega_{m n k}^{3}} \sin \omega_{m n k} \Delta t\right]\right\} \cdot f_{i} \\
& +\delta_{p} \cdot \sum_{i=1}^{p-1}\left\{\sin \omega_{m n k} \Delta t(p-i)\left[\frac{4}{\Delta t \omega_{m m k}^{3}}\left(2+\cos \omega_{m n k} \Delta t\right)-\frac{12}{\Delta t^{2} \omega_{m n k}^{4}} \sin \omega_{m n k} \Delta t\right]\right\} \cdot \bar{f}_{i},
\end{aligned}
$$

where $\delta_{p}$ has been defined earlier. From (57), (60), (43) and (58) we have:

and

$$
a_{p} f_{p}^{2 / 3}+b_{p} f_{p}+c_{p} \bar{f}_{p}+d_{p}=0
$$

$$
\bar{a}_{p} \bar{f}_{p} \cdot f_{p}^{-1 / 3}+\bar{b}_{p} f_{p}+\bar{c}_{p} \bar{f}_{p}+\bar{d}_{p}=0
$$

where the coefficients $a_{p}, b_{p}, \ldots, \bar{d}_{p}$ are given in appendix $\mathrm{E}$ in Nosier et al (1992). Introducing $f_{p}=g_{p}^{3},(61 \mathrm{~b})$ becomes:

$$
h_{4 p} \cdot g_{p}^{4}+h_{3 p} g_{p}^{3}+h_{2 p} g_{p}^{2}+h_{1 p} g_{p}+h_{o p}=0 .
$$

The expressions for $h_{j p}(j=0,1, \ldots, 4)$ in terms of $a_{p}, b_{p}, \ldots, \bar{d}_{p}$ are displayed in appendix $\mathrm{E}$ in Nosier et al (1992).

Upon solving (62) for $g_{p}$ (for example by the Newton-Raphson method), $f_{p}$ and $\bar{f}_{p}$ can be determined for every $p=1,2, \ldots$. The impactor position at any time $t=p \Delta t$ is obtained by:

$$
z(p \Delta t)=v_{o}(p \Delta t)-\frac{3}{20} \frac{(\Delta t)^{2}}{m_{0}} f_{p}+\frac{1}{30} \frac{(\Delta t)^{3}}{m_{0}} \bar{f}_{p}-\delta_{p} \cdot \sum_{i=1}^{p-1} \frac{(\Delta t)^{2}}{m_{0}}\left[(p-i) f_{i}-\frac{\Delta t}{15} \bar{f}_{i}\right]
$$

The displacement of the plate at the contact point $\left(x=a_{0}, y=b_{0}\right)$ according to various plate theories is obtained by substituting $(60 \mathrm{~b})$ into $(31)$ :

$$
\begin{aligned}
& u_{3}\left(a_{0}, b_{0},-h / 2, p \Delta t\right)= \\
& \quad \sum_{m=1}^{\infty} \sum_{n=1}^{\infty} \sum_{k=1}^{k} \bar{B}_{m n k}\left\{\left[\frac{1}{\omega_{m n k}}-\frac{12}{\Delta t^{3} \omega_{m n k}^{4}} \sin \omega_{m n k} \Delta t+\frac{6}{\Delta t^{2} \omega_{m n k}^{3}}\left(1+\cos \omega_{m n k} \Delta t\right)\right] f_{p}\right. \\
& +\left[\frac{6}{\Delta t^{2} \omega_{m n k}^{4}} \sin \omega_{m n k} \Delta t-\frac{2}{\Delta t \omega_{m n k}^{3}}\left(2+\cos \omega_{m n k} \Delta t\right)\right] \bar{f}_{p} \\
& +\delta_{p} \sum_{i=1}^{p-1}\left\{\sin \omega_{m n k} \Delta t(p-i)\left[\frac{24}{\Delta t^{3} \omega_{m n k}^{4}}\left(1-\cos \omega_{m n k} \Delta t\right)-\frac{12}{\Delta t^{2} \omega_{m n k}^{3}} \sin \omega_{m n k} \Delta t\right]\right\} f_{i} \\
& +\delta_{p} \sum_{i=1}^{p-1}\left\{\cos \omega_{m n k} \Delta t(p-i)\left[\frac{12}{\Delta t^{2} \omega_{m n k}^{4}} \sin \omega_{m n k} \Delta t-\frac{4}{\Delta t \omega_{m n k}^{3}}\left(2+\cos \omega_{m n k} \Delta t\right]\right\} \bar{f}_{i}\right\}
\end{aligned}
$$

where, as before, $K=3(N+1)$ in LWPT, $K=5$ in FSDPT and TSDPT, and $K=3$ in CLPT. 


\subsection{Determination of impact force using loading VI}

Here we consider the determination of the total impact force according to the loading model VI. The transverse displacement of the laminate at the impact point according to various theories is determined from (35) and (37). Substituting these equations and (32) and (41) into (43) results in

$$
\begin{aligned}
& K_{2}^{-2 / 3} F^{2 / 3}(t)=v_{o} t-\left(1 / m_{0}\right) \int_{0}^{t} F(\tau)(t-\tau) \mathrm{d} \tau-\sum_{m=1}^{\infty} \sum_{n=1}^{\infty} \sum_{k=1}^{K} \bar{B}_{m n k} \\
& \quad \times \int_{0}^{t}\left\{\sin \left[\gamma_{m n} F^{1 / 3}(\tau)\right]-\gamma_{m n} F^{1 / 3}(\tau) \cos \left[\gamma_{m n} F^{1 / 3}(\tau)\right]\right\} \sin \omega_{m n k}(t-\tau) \mathrm{d} \tau,
\end{aligned}
$$

where $\gamma_{m n}$ has been defined earlier. The total force $F(t)$ in (65) can be replaced by either $\alpha(t)$ with the help of (32) or by the radius of contact region $R_{o}(t)$ with the help of (34). In either case, the nonlinear integral equation (65) may not have an analytical solution. Therefore, only a numerical solution of this equation will be presented here. Assuming a linear variation for the total force $F(t)$ during each small time increment, the first integral in (65) at time $t=p \Delta t(p=1,2, \ldots)$ is given as in (49a). To evaluate the second integral appearing in (65) we assume that the total force $F(t)$ is constant during each small time increment. That is, for $(i-1) \Delta t \leqslant t \leqslant i \Delta t$; we assume that

$$
F(t)=\frac{1}{2}\left(f_{i}+f_{i-1}\right)=\tilde{f}_{i},
$$

where, as before, $f_{i}$ is the magnitude of the force $F(t)$ at time $t=i \Delta t$. The variable $\tilde{f}_{i}$ is introduced for simplicity. The second integral in (65) will be

$$
\begin{gathered}
\int_{0}^{p \Delta t}\left\{\sin \left[\gamma_{m n} F^{1 / 3}(\tau)\right]-\gamma_{m n} F^{1 / 3}(\tau) \cos \left[\gamma_{m n} F^{1 / 3}(\tau)\right]\right\} \cdot \sin \omega_{m n k}(p \Delta t-\tau) \mathrm{d} \tau \\
=\frac{1}{\omega_{m n k}}\left(1-\cos \omega_{m n k} \Delta t\right)\left[\sin \left(\gamma_{m n} \tilde{f}_{p}^{1 / 3}\right)-\gamma_{m n} \tilde{f}_{p}^{1 / 3} \cdot \cos \left(\gamma_{m n} \tilde{f}_{p}^{1 / 3}\right)\right] \\
+\frac{\delta_{p}}{\omega_{m n k}} \sum_{i=1}^{p-1}\left[\cos \omega_{m n k}(p-i) \Delta t-\cos \omega_{m n k}(p-i+1) \Delta t\right. \\
\times\left[\sin \left(\gamma_{m n} \tilde{f}_{i}^{1 / 3}\right)-\gamma_{m n} \tilde{f}_{i}^{1 / 3} \cos \left(\gamma_{m n} \tilde{f}_{i}^{1 / 3}\right)\right]
\end{gathered}
$$

Upon substitution of (49a), and (67) into (65) we obtain

$$
\begin{aligned}
& a_{p}\left(2 \tilde{f}_{p}-f_{p-1}\right)^{2 / 3}+b_{p} \tilde{f}_{p}+c_{p} \\
& +\sum_{m=1}^{\infty} \sum_{n=1}^{\infty} \sum_{k=1}^{K} \frac{\bar{B}_{m n k}}{\omega_{m n k}}\left(1-\cos \omega_{m n k} \Delta t\right) \\
& \quad \times\left[\sin \left(\gamma_{m n} \tilde{f}_{i}^{1 / 3}\right)-\gamma_{m n} \tilde{f}_{p}^{1 / 3} \cdot \cos \left(\gamma_{m n} \tilde{f}_{p}^{1 / 3}\right)\right]=0,
\end{aligned}
$$

where $a_{p}, b_{p}$, and $c_{p}$ are given in Nosier et al (1992).

Upon numerical solution of (68) the value of $\tilde{f}_{p}$ at $t=p \Delta t$ and, therefore, the value of $f_{p}$, will be obtained. The impactor position is determined from (63). The transverse displacement of the laminate at the point of impact according to various theories at time $t=p \Delta t$ is obtained by substituting (67) into (36) and (37). 


\section{Numerical results and discussions}

Throughout our numerical examples we assume that the impact occurs at the middle of the plate (or beam). That is $a_{0}=a / 2$ and $b_{0}=b / 2$.

\section{Example 1}

Timoshenko (1913) considered the problem of a simply-supported isotropic beam $(1 \times 1 \times 30.7 \mathrm{~cm})$ subjected to the transverse impact of a $2-\mathrm{cm}$ radius steel ball with an initial velocity equal to $1 \mathrm{~cm} / \mathrm{s}$. The time histories of the total impact force $F(t)$, the impactor position, the beam deflection, the indentation $\alpha(t)$, and the in-plane stress at the outer fibre of the beam are given in Nosier et al (1992). In Timoshenko's solution the response quantities are obtained up to time $t \simeq 1778 \times 10^{-6} \mathrm{~s}$ and therefore only two collisions were observed. The present results, on the other hand, indicate that a third collision also occurs at $t \simeq 1800 \times 10^{-6} \mathrm{~s}$. For this problem, the indentation is small indicating that the contact region is very small. From the numerical results it was concluded that (i) the impact force can be accurately determined by considering a fairly reasonable number of normal modes, and (ii) the accurate determination of stress requires the superposition of more normal modes which can drastically increase the computer execution time. In obtaining the present results, the impact load was represented as a concentrated load (case-I loading model). Also the nonlinear integral equation (43) was solved using the linear Lagrange interpolation functions with a time increment $\Delta t=10(\mu \mathrm{s})$. To check the accuracy of our results, we also solved (43) using the Hermite cubic polynomials. It was observed that when the linear Lagrange functions are used, the solution remains accurate and stable for a wide range of $\Delta t$ when the impact response of the structure is obtained analytically.

\section{Example 2}

In this example we consider the impact response of a ten-layered symmetric laminate $\left(0^{\circ} / 90^{\circ} / 0^{\circ} / 90^{\circ} / 0^{\circ}\right)_{s}$, which is also considered by Qian \& Swanson (1980), with material properties: $E_{11}=120 \mathrm{GPa}, E_{22}=7.9 \mathrm{GPa}, G_{12}=G_{13}=5.5 \mathrm{GPa}, v_{12}=v_{23}=0.3$, $\rho=1580 \mathrm{~kg} / \mathrm{m}^{3}, a=b=0.2 \mathrm{~m}$. The laminate is impacted by a $12.7 \mathrm{~mm}$ diameter steel ball $\left(\rho=7960 \mathrm{~kg} / \mathrm{m}^{3}\right)$ with an initial velocity $v_{0}=3 \mathrm{~m} / \mathrm{s}$. It was assumed that the impactor is rigid so that the Hertzian contact coefficient $K_{2}$ can be obtained from $K_{2}=(4 / 3) \sqrt{R_{S}} E_{22}$. This way it is found that $K_{2}=8.394 \times 10^{8} \mathrm{~N} / \mathrm{m}^{3 / 2}$. Qian \& Swanson (1990) developed numerical results, based on FSDPT with the shear correction factors equal to $\pi^{2} / 12$, by using two different techniques. Their first technique was based on the Rayleigh-Ritz method, with numerical integration in time, and the Hertzian contact law. In their second technique they replaced the Hertzian contact law, by a linear contact law

$$
F=K_{1} \propto .
$$

Therefore, instead of a nonlinear integral equation they analytically solved a linear 
integral equation similar to

$$
\begin{aligned}
K_{1}^{-1} F(t)=v_{0} t- & \frac{1}{m_{0}} \int_{0}^{t} f(\tau) \cdot(t-\tau) \mathrm{d} \tau \\
& -\sum_{m=1}^{\infty} \sum_{m=1}^{\infty} \sum_{k=1}^{K} \bar{B}_{m n k} \int_{0}^{t} f(\tau) \cdot \sin \omega_{m n k}(t-\tau) \mathrm{d} \tau,
\end{aligned}
$$

by using the Laplace transformation technique. In so doing, they had to find the infinite roots of a polynomial equation by using a numerical scheme. Rather than solving (70) analytically as in Qian \& Swanson (1990), if we follow our procedure and use the global linear Lagrange interpolation functions for the approximation of the total contact force $F(t)$, we obtain

$$
\left(K_{1}^{-1}+b_{p}\right) \cdot f_{p}+c_{p}=0
$$

which yields

$$
f_{p}=-c_{p} /\left(b_{p}+K_{1}^{-1}\right)
$$

where $b_{p}$ and $c_{p}$ are the same as defined earlier. It is our belief that the solution (72) is more direct and less involved than the analytical one proposed by Qian \& Swanson (1990). In that paper it was assumed that $k_{1}=5.866 \times 10^{6} \mathrm{~N} / \mathrm{m}$ and numerical results based on the Hertzian and the linear contact laws were compared. Three different

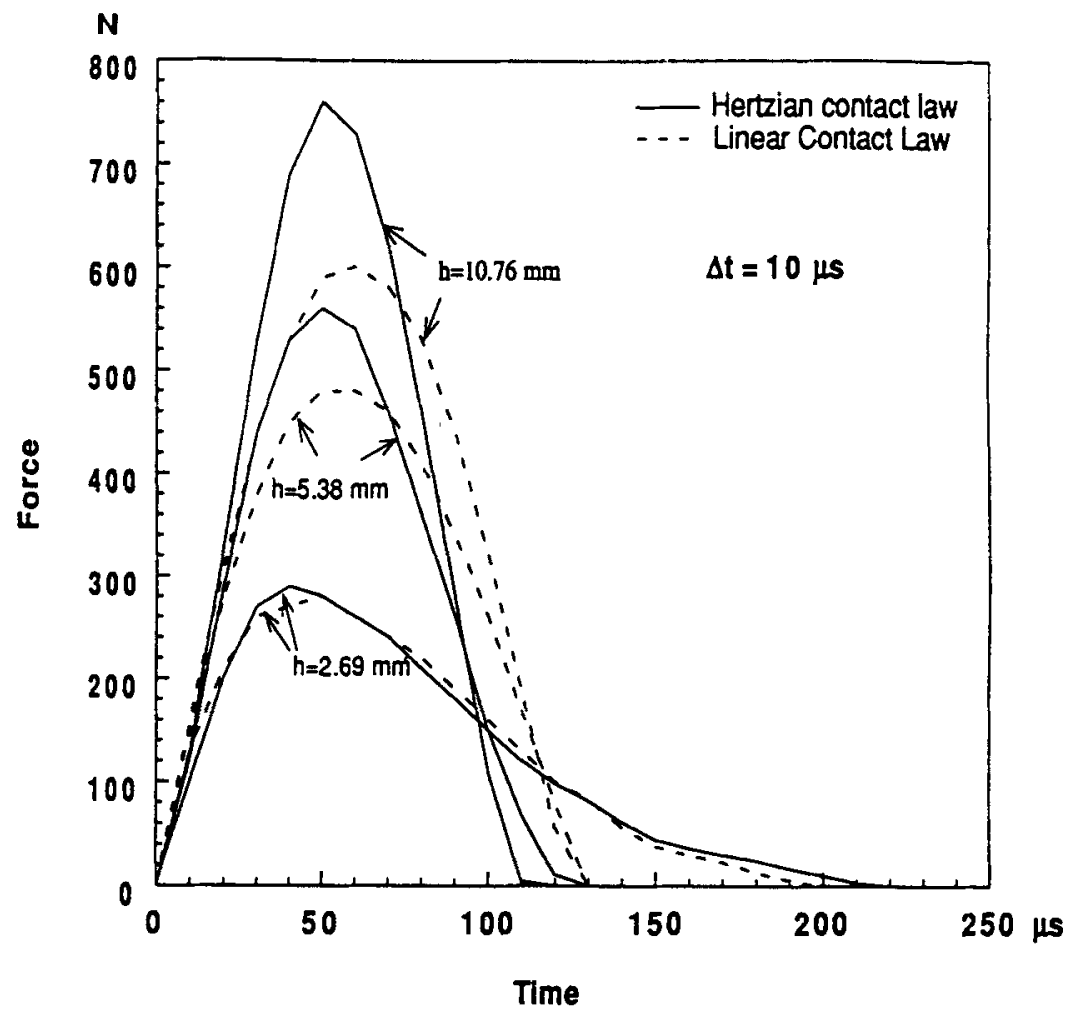

Figure 5. The contact force history of $\left(0^{\circ} / 90^{\circ} / 0^{\circ} / 90^{\circ} / 0^{\circ}\right)_{s}$ laminate. 
thicknesses were assumed for the laminate. It was assumed that the impact load was distributed uniformly over a small square region. To check our analyses and computer codes, we developed the numerical results of figure 5 in that paper which are displayed here in figure 5. The results of Qian \& Swanson (1990) are not presented here since they are identical to our results. It is clear from figure 5 that Hertzian and linear contact laws yield almost identical results for a thin plate. This, of course, is due to the fact that the significance of indentation vanishes for thin plates. This conclusion was also reached by Qian \& Swanson (1990). It is to be remembered that no clear and scientific methodology yet exists for determination of the contact coefficient $k_{1}$ of the linear law.

In order to obtain an assessment concerning the significance of various loading models considered in this report, we have displayed in figure 6 the time history of the impact force of the ten-layered laminate (with $h=2.69 \mathrm{~mm}$ ). The impact load in figure 6 is modelled according to cases I and VI loading models. Remember that in the case VI loading model the load is assumed to be distributed in accordance to the Hertzian contact law and, furthermore, the time-dependence of the contact region is taken into account. It is seen from figure 6 that the two models yield identical results for a reasonably small $\Delta t$. The results of two models are not, of course, identical when a larger time increment is assumed. This is primarily due to the fact that in the numerical evaluation of the second integral appearing in equation (65) we assumed that the contact force remains constant during each small time increment. Our conclusion here is that since the contact region is often an extremely small region,

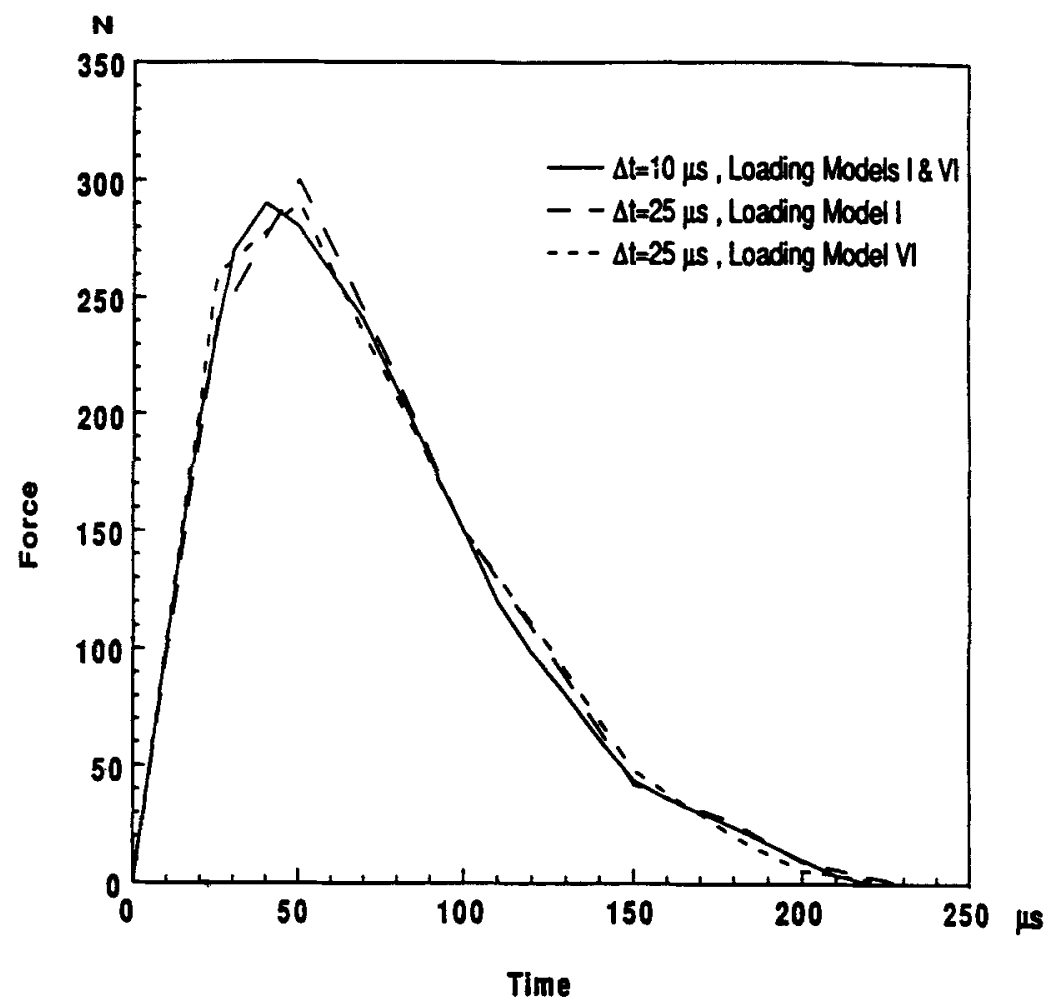

Figure 6. Comparison of contact force predicted by various loading models. 
the loading model I will yield very accurate results as far as the total impact force $F(t)$ and the transverse displacement of the laminate are concerned. This is particularly true when the equivalent single-layer theories are employed, since transverse inextensibility is assumed in such theories. However, it is found, based on the results of LWPT, that a realistic description for both stresses in the vicinity of the contact region and the impact force is only obtained from the case-VI loading model. The calculated values of the total force at various time steps can then be used to obtain the corresponding values of the radius of the circular contact region.

The convergence study of the in-plane stress $\sigma_{11}$ (see Nosier et al 1992 for details regarding the stress calculations), at the point of contact $(x=a / 2, y=b / 2, z=-h / 2)$ is accomplished with the help of figure 7 . The results indicate that a relatively large number of normal modes are required to achieve convergence for the stress. It will soon become clear that a correct distribution for stresses near the contact region cannot be obtained from any of the single-layer theories during the contact period.

When $h=2.69 \mathrm{~mm}$ multiple (two) impacts occur in figures 5 and 6 , but only the first impact is shown here. This problem was also considered by Sun \& Chen (1985) who used a plastic contact law and an experimentally determined value for $K_{2}=1.413 \times 10^{9} \mathrm{~N} / \mathrm{m}^{3 / 2}$. Using this value, and based on FSDPT, we have displayed the time history of the impact force in figures 8 and 9 . Comparison of figures 7 and 8 reveals that, as opposed to stress calculation, a relatively low number of normal modes is required for the convergence of the impact force. This conclusion is also valid as far as the transverse displacement is concerned. We should emphasize at this

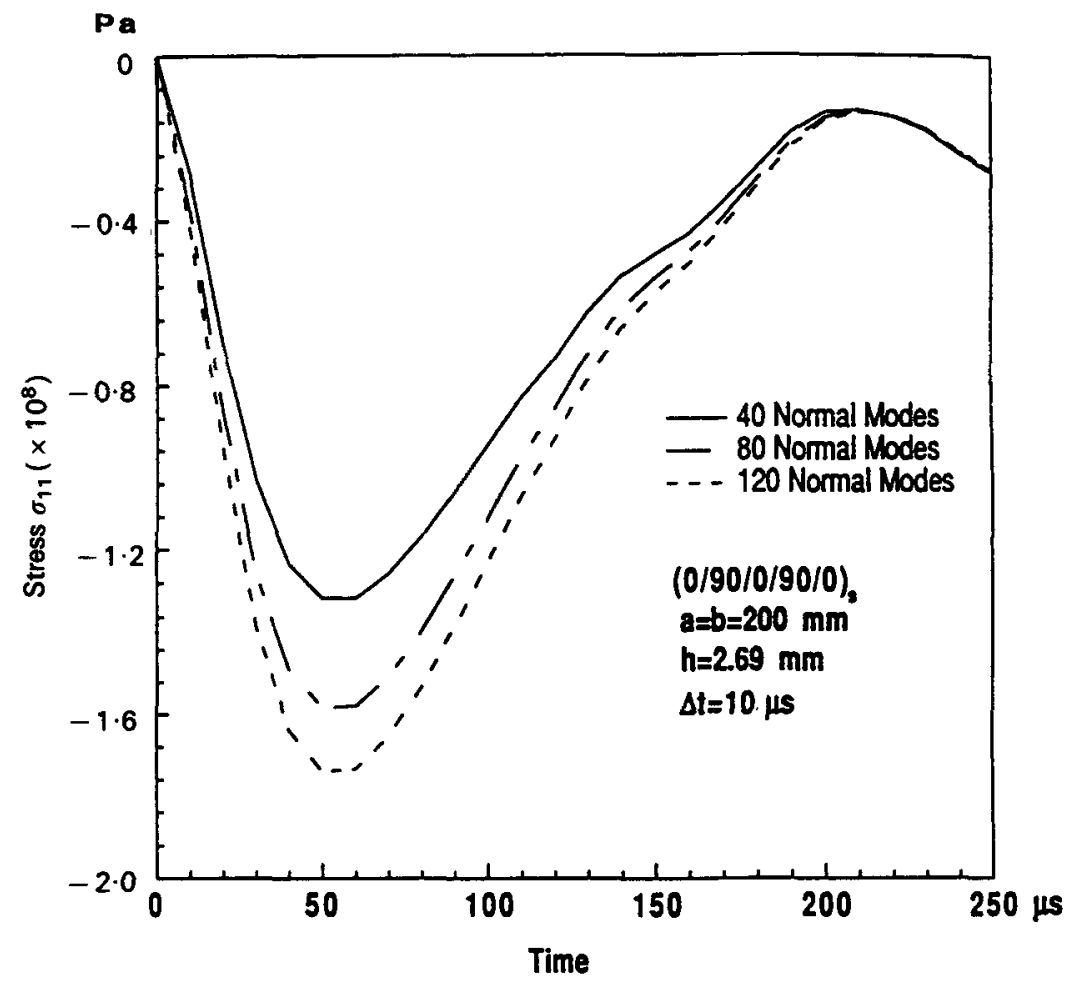

Figure 7. Convergence study for the in-plane stress $\sigma_{11}$ at the contact point $(x=a / 2, y=b / 2, z=-h / 2)$. 


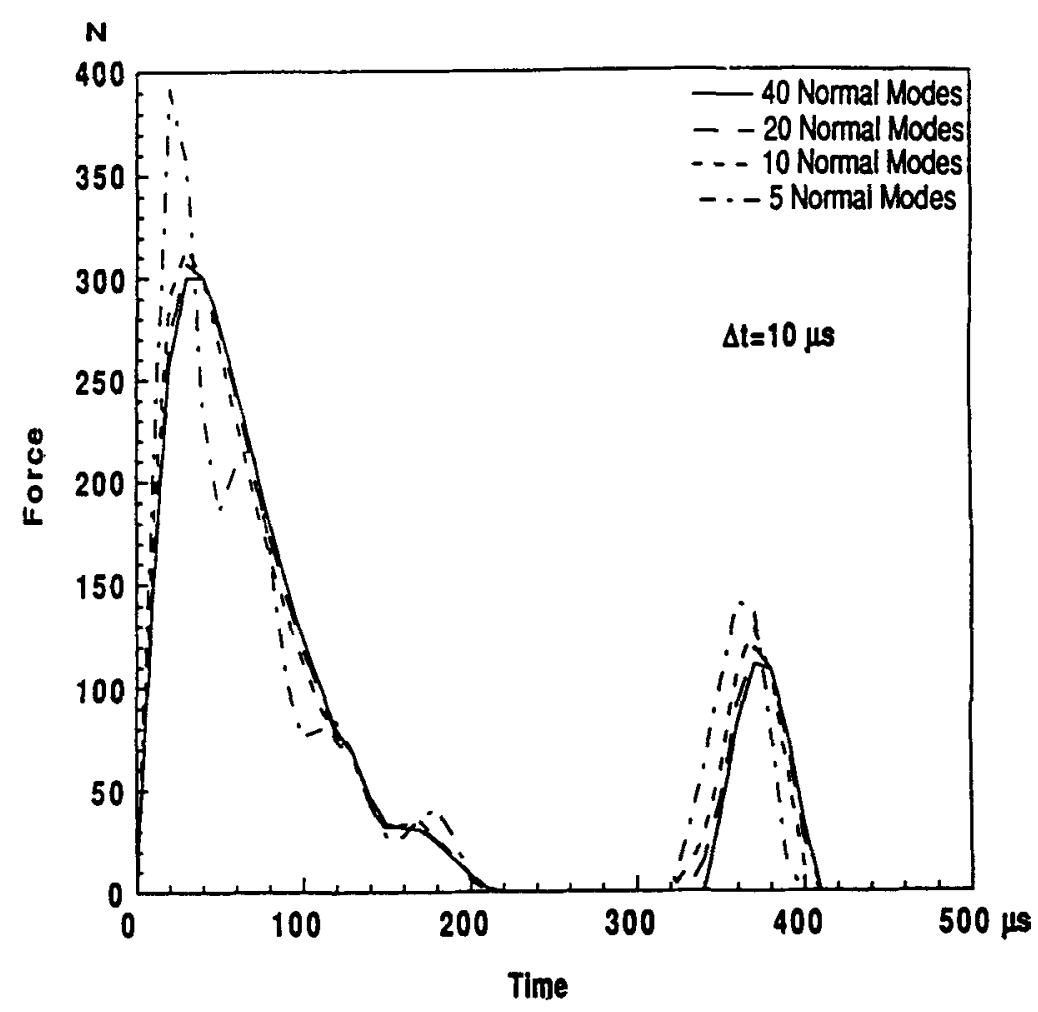

Figure 8. Convergence study for the contact force.

point that this rapid convergence is a direct consequence of the transverse inextensibility (in the thickness direction) assumption in the equivalent single-layer theories. We will demonstrate that the convergence of the impact force and the displacement at the point of contact is quite slow by using LWPT. Furthermore, it will become apparent that none of the response quantities in the vicinity of the contact region is correctly predicted by the equivalent single-layer theories during the contact period. The results of figure 9 also indicate that the analytical solution remains stable and accurate for relatively large values of $\Delta t$.

\section{Example 3}

Here we consider a three-layered laminate $\left(0^{\circ} / 90^{\circ} / 0^{\circ}\right)$ with material properties: $E_{1}=25.1 \times 10^{6} \mathrm{psi}, E_{2}=4.8 \times 10^{6} \mathrm{psi}, E_{3}=0.75 \times 10^{6} \mathrm{psi}, G_{12}=1.36 \times 10^{6} \mathrm{psi}, G_{13}=$ $1.2 \times 10^{6} \mathrm{psi}, G_{23}=0.47 \times 10^{6} \mathrm{psi}, v_{12}=0.036, v_{13}=0.171, \rho=1.4667 \times 10^{4} \mathrm{lb} \cdot \mathrm{s}^{2} / \mathrm{in}^{4}$. We will use the case-VI loading model in the remaining developments. We assume that the square plate $(a=b=10 \mathrm{in}$.) is impacted by a $0.25 \mathrm{in}$. radius steel impactor with a mass equal to $4.8 \times 10^{-5} \mathrm{lb} \cdot \mathrm{s}^{2} / \mathrm{in}$. and an initial velocity of $100 \mathrm{in} . / \mathrm{s}$. As we pointed out earlier, the contact coefficient $K_{2}$ appearing in the Hertz law must be obtained experimentally, at least for orthotropic laminates. Here by using the modified Hertz law we find that $K_{2}=2 \cdot 791 \times 10^{6} \mathrm{lb} / \mathrm{in}^{3 / 2}$. If we follow the procedure suggested by Greszczuk (1982), we obtain $K_{2}=0.837 \times 10^{6} \mathrm{lb} / \mathrm{in}^{3 / 2}$. In evaluating $K_{2}$, we also assumed that $v_{z}=\frac{1}{2}\left(v_{13}+v_{23}\right)$ and $G_{z}=\frac{1}{2}\left(G_{13}+G_{23}\right)$. Clearly there exists a big 


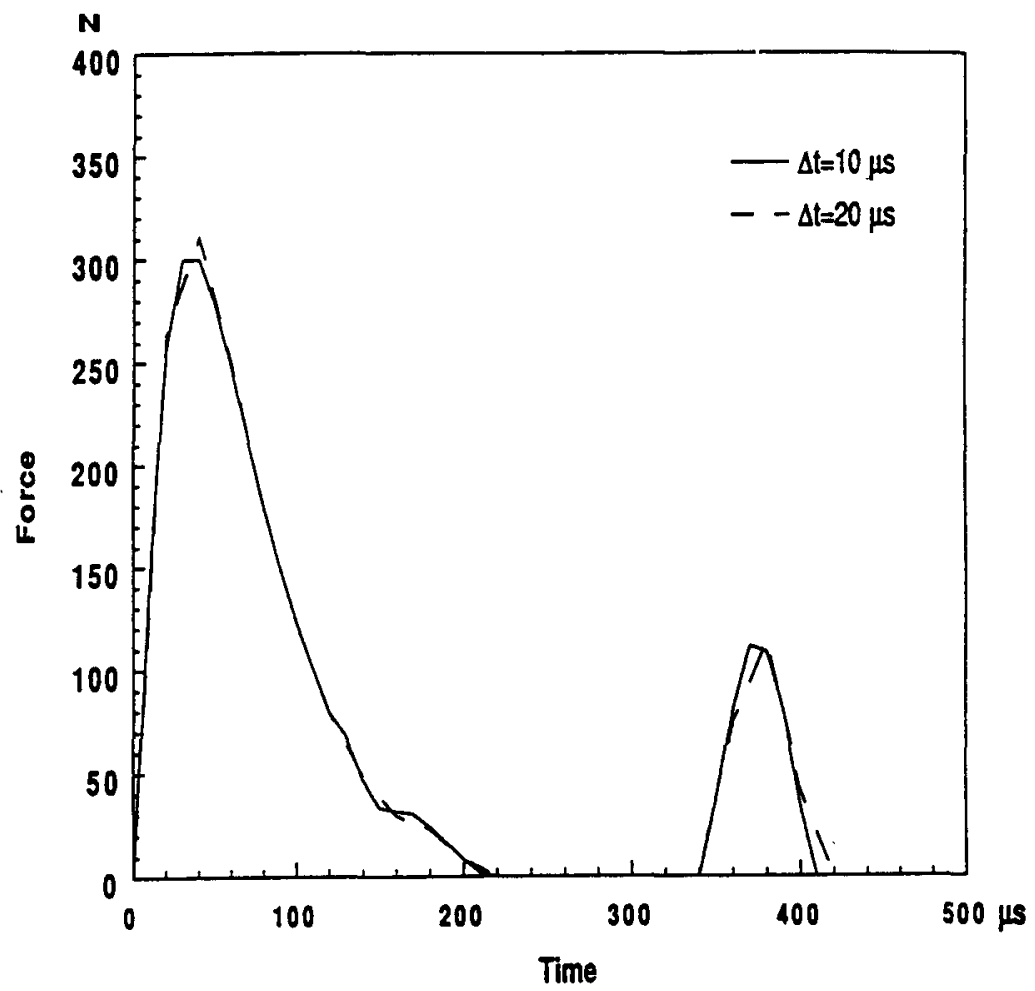

Figure 9. The effect of time increment $\Delta t$ on the contact force history.

difference between the two values of $K_{2}$. Most likely the actual value of $K_{2}$ lies somewhere between these two values. This conclusion is merely based on the observation that the value of the out-of-plane Young's modulus $E_{3}$ is much smaller than $E_{2}$. Here, however, for numerical calculations we use the value of $K_{2}=2.791 \times 10^{6} \mathrm{lb} / \mathrm{in}^{3 / 2}$. Also, we again use $\pi^{2} / 12$ for the shear correction factors in FSDPT. We have displayed the time-histories of the impact force and the transverse displacement of the laminate $(a / h=40)$ in figures 10 and 11 , respectively. In developing the numerical results based on LWPT we modelled each physical layer as two layers. Figure 10 is clear justification for employing this theory in the impact problems. The inaccuracy of the equivalent single-layer theories in such problems stems from the fact that the assumptions of indentation and transverse inextensibility are made simultaneously. Indeed, from figure 11 it is observed that during contact there is a significant difference between the transverse displacements at the point of contact $(x=a / 2, y=b / 2, z=-h / 2)$ and at the point $(x=a / 2, b / 2, z=h / 2)$. After the contact period the two displacements become essentially identical. The slight differences cannot be distinguished from the figures. For clarity, the time-histories of the displacement of the plate at the contact point, the impactor position, and indentation during contact period are displayed in figure 12 .

Similar results are also generated for a thicker plate $(a / h=20)$ and are shown in figures 13 and 14. It is observed that for such a plate there is yet a more significant difference between the transverse displacements at the contact point and at point 


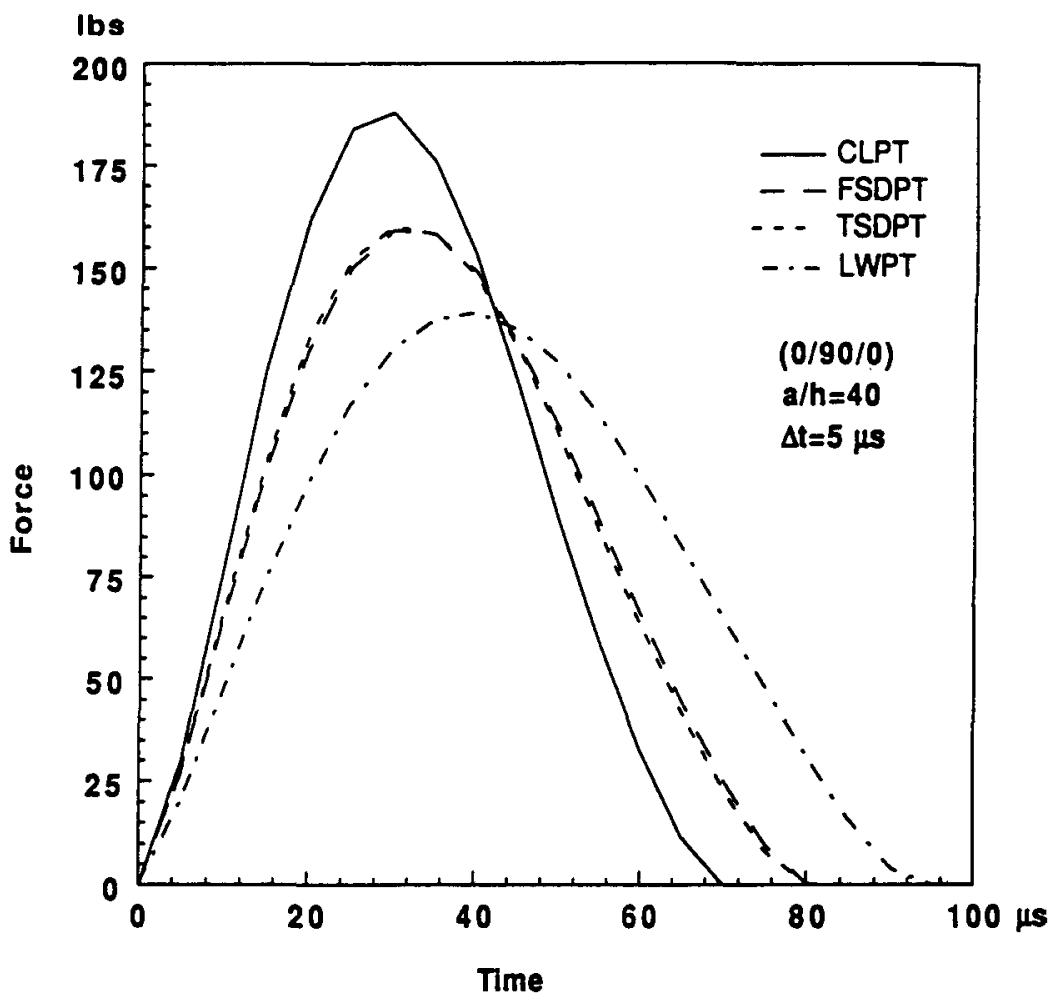

Figure 10. Comparison of the contact force histories predicted by various theories.

$(x=a / 2, y=b / 2, z=h / 2)$. This is the primary reason for the impact force being lower than that predicted by the equivalent single-layer theories.

Results for an additional example, related to impact response of an antisymmetric cross-ply laminate $\left(0^{\circ} / 90^{\circ}\right)$ are given in Nosier et al (1992).

\section{Summary and conclusions}

In this report we have developed the low-velocity impact dynamic analyses of a laminated plate according to four different plate theories. The first three theories belong to the class of the equivalent single-layer theories in which the transverse extensibility of the plate is ignored. The fourth theory, the one which takes this effect into account by representing the displacements in a layerwise manner, is called the layerwise plate theory of Reddy (LWPT). Throughout this study we have demonstrated the effectiveness of this theory through several numerical examples pertaining to the natural frequencies, the impact force, the displacement, and the in-plane and interlaminar stress components (Nosier et al 1992). In particular, we have found that the rate of convergence of all the response quantities, including the impact force and the transverse displacement, is basically the same and very slow, specially during the contact period and in the vicinity of the contact zone, according to the layerwise theory. More importantly, a full three-dimensional description of the stress field is 


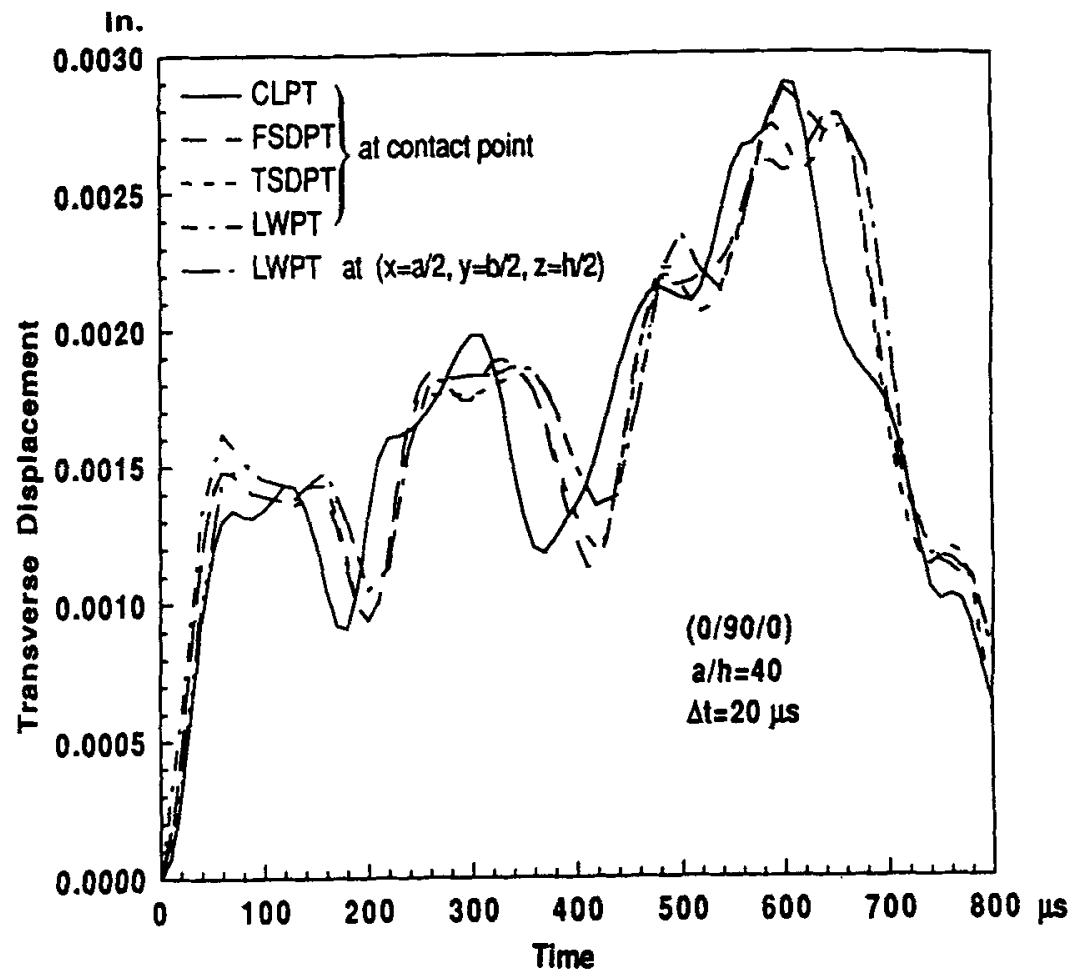

Figure 11. Comparison of the displacement of a laminate at the contact point and at point $(x=a / 2, y=b / 2, z=h / 2)$.

obtained only through LWPT. The results of this theory indicate a tremendous amount of stress concentration during the contact period in the vicinity of the contact point. During the contact period, the local deformation in the thickness direction in the neighbourhood of the contact region is the primary reason for the stress build-ups. On the other hand, the nature of the stress is mainly flexural in the free-vibration region. Indeed, for a thick plate the flexural stress is negligible as compared with the infinitely large values of the stress components in the contact region during the duration of the contact. All these observations were made through the employment of the layerwise theory. In fact, only now through the use of LWPT, not through a single-layer-theory, can the matrix cracking, fibre breakage, debonding, and other experimentally observed phenomena be explained. To predict the onset of any failure one should incorporate a failure criterion into our impact analysis. Because of the full 3-D capacity of LWPT, we more realistically modelled the impact pressure in accordance with the Hertzian law of contact. This model, named in this paper as the case-VI loading model, takes into account the time-dependence of the contact area. This, on the other hand, has necessitated the solution of a relatively more complicated nonlinear integral equation. In solving this equation, we made the assumption that the impact force was constant during small time intervals in evaluating the second integral appearing in this equation. In order to be able to use a larger time step in the analysis, the second integral must be, as in case of the first integral, evaluated by assuming a linear variation for the impact force during various time intervals. 
In.

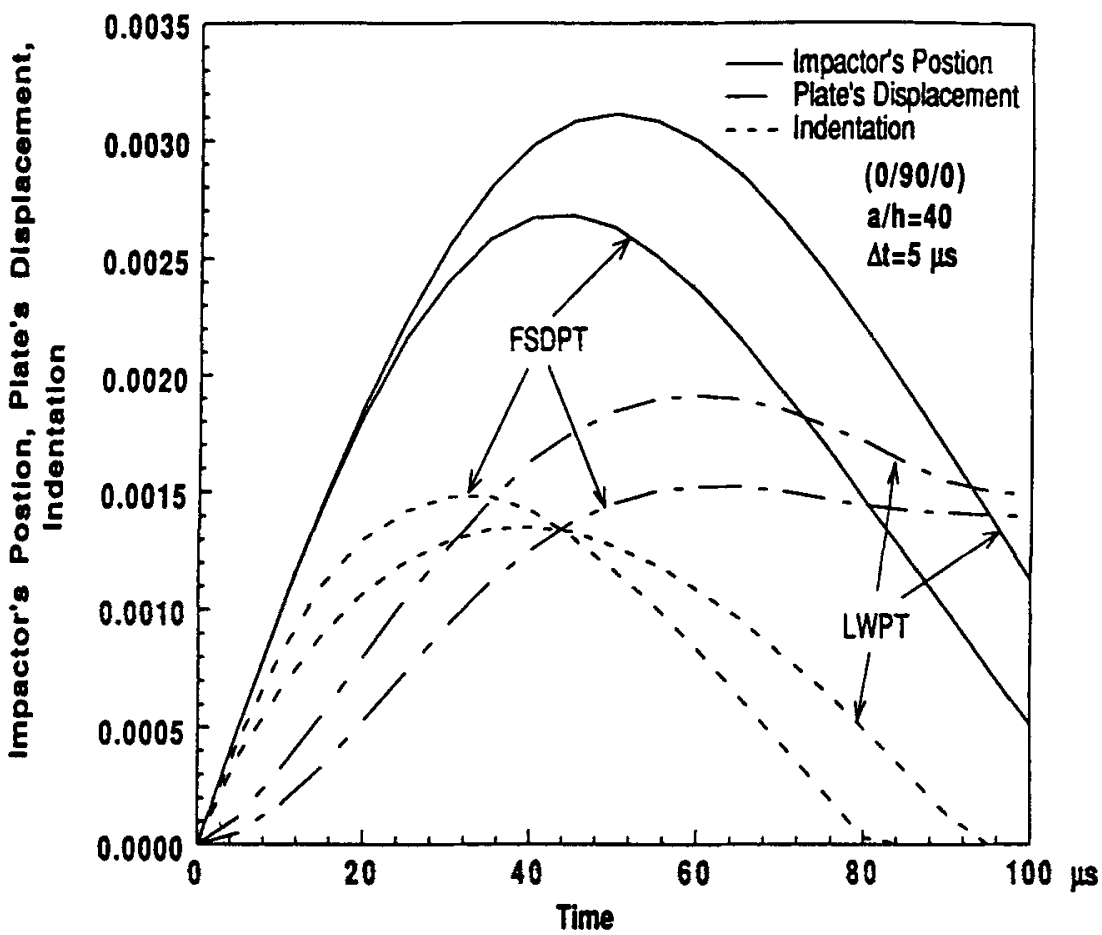

Figure 12. Comparison of the laminate displacement, indentation, and impactor's pesition at the contact point $(x=a / 2, y=b / 2, z=-h / 2)$.

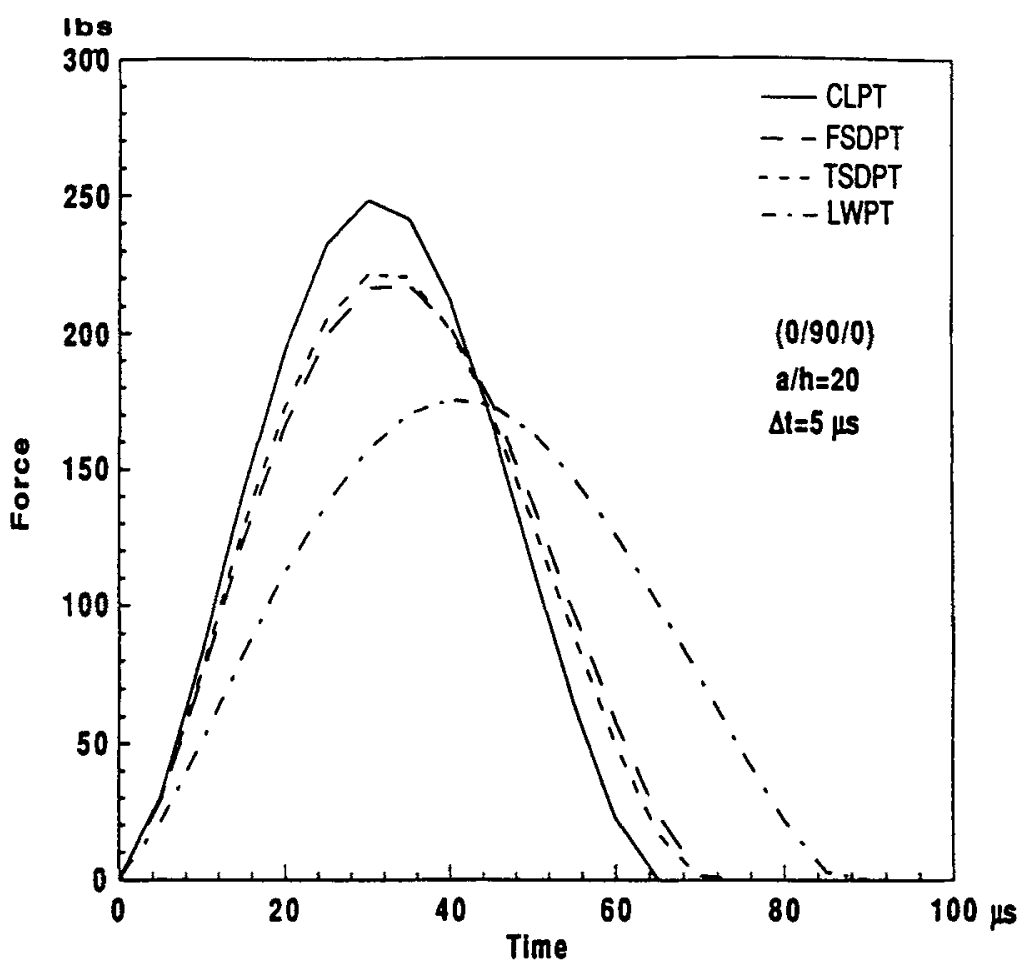

Figure 13. The contact force history of a $\left(0^{\circ} / 90^{\circ} / 0^{\circ}\right)$ laminate $(a / h=20)$ according to various theories. 
In.

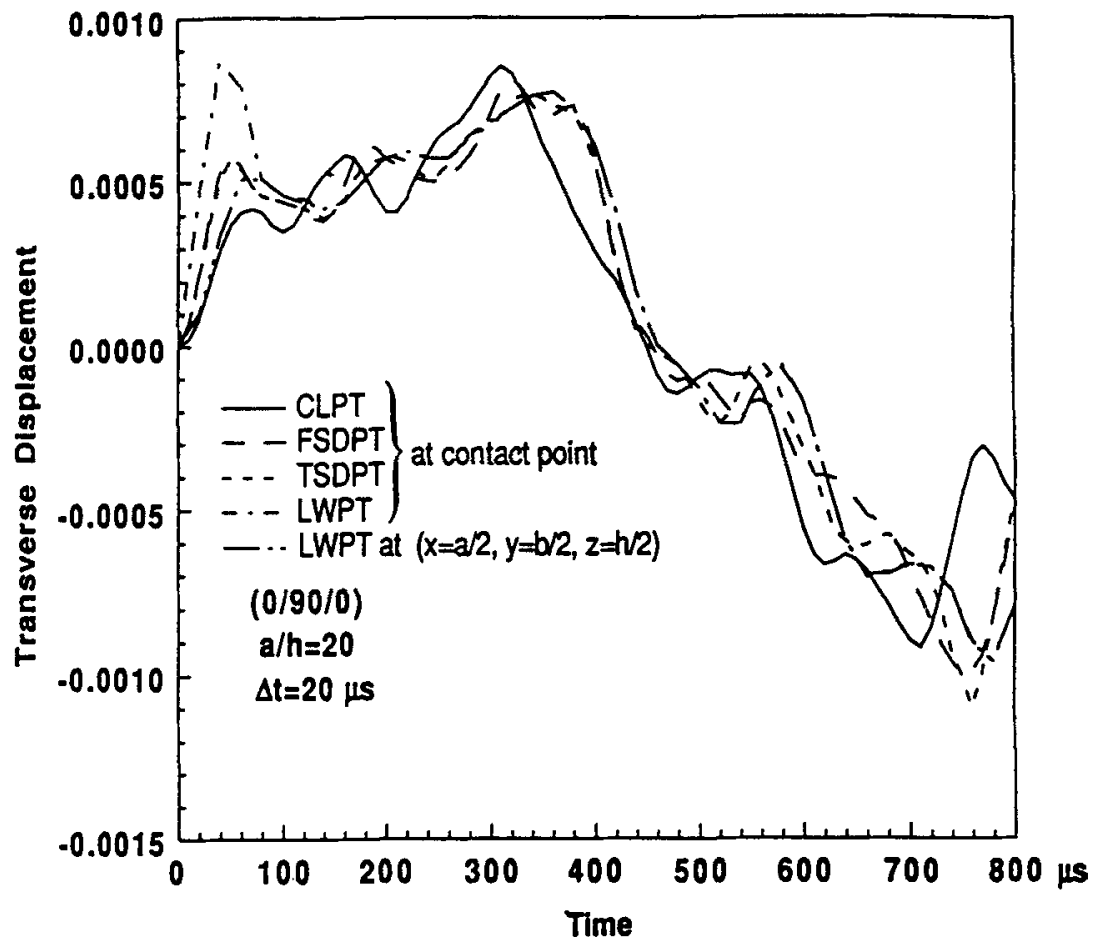

Figure 14. The transverse displacement of $\left(0^{\circ} / 90^{\circ} / 0^{\circ}\right)$ laminate $(a / h=20)$ according to various theories.

As far as the equivalent single-layer theories are concerned, we point out that the response quantities predicted by these theories, especially by the shear-deformation theories, are accurate to a certain degree only in the free-vibration period. During the contact period, the results of these theories are valid only at points away from the contact region. On the other hand, we are often only concerned with a precise description of the stress field in the contact zone neighbourhood because of the existence of high stress concentrations. For this reason the usefulness of the equivalent single-layer theories is questionable.

\section{References}

Abrate S 1991 Impact on laminated composite materials. Appl. Mech. Rev. 44: 155-190 Bogdanovich A E, Yarve E V 1989 Numerical analysis of laminated plates subjected to impact loading. Proc. of the American Society for Composites, 4th Technical Conference (Blacksburg, VA: Virginia Polytechnic Inst.) pp. 399-409

Bogdanovich A E, Yarve E V 1990 Calculation of damage zones in laminated composite plates subjected to low velocity impact. Proc. of the American Society for Composites, 5th Technical Conference, East Lansing, Michigan, pp 1-11

Byun C, Kapania R K 1991 Prediction of interlaminar stresses in laminated plates using global orthogonal polynomials. Enhanced analysis techniques for composite materials (eds) L Schwer, J N Reddy, A Mal, ASME Winter Annual Meeting, Atlanta, pp. 113-124 
Byun C, Kapania R K 1992 Nonlinear impact response of thin imperfect laminated plates using a reduction method. Compos. Eng., Int. J. 2: 391-410

Cairns D S, Lagace P A 1989 Transient response of graphite epoxy and kevlar epoxy laminates subjected to impact. $A I A A$ J. 27: 879-884

Chaudhuri R A, Seide P 1987 An approximation semi-analytical method for prediction of interlaminar shear stresses in arbitrary laminated thick plates. Comput. Struct. 25: 627-636

Chen J K, Sun C T 1985 Dynamic large deflection response of composite laminates subjected to impact. Compos. Struct. 4: 59-73

Conwag H D 1956 The pressure distribution between two elastic bodies in contact. Z. Angew. Math. Phys. 7: 460-465

Goldsmith W 1960 Impact: The theory and physical behaviour of colliding solids (London: Edward Arnold)

Greszczuk L B 1975 Response of isotropic and composite materials in particle impact. Am. Soc. Test. Mater., Spec. Tech. Publ. 568: 183-211

Greszczuk L B 1982 Damage in composite materials due to low velocity impact. In Impact dynamics (eds) J A Zukas, T Nicholas, H F Swift, L B Greszczuk, D R Curran (New York: John Wiley)

Kapania R K, Raciti S 1989 Recent advances in analysis of laminated beams and plates. Part II: Vibrations and wave propagation. AlAA J. 27: 935-949

Kapania R K, Yang T Y 1986 Formulation of an imperfect quadrilateral doubly-curved shell element for post-buckling analysis. AIAA J. 24: 310-311

Kant T, Mallikarjuna 1991 Nonlinear dynamics of laminated plates with a higher-order theory and $C^{0}$ finite elements. Int. J. Nonlinear Mech. 26: 335-343

Nosier A, Kapania R K, Reddy J N 1992 Low velocity impact response of laminated plates. Center for Composite Materials and Structures, Report 92-20, vPI \& sU, Blacksburg, VA

Obst A W, Kapania R K 1992 Nonlinear static and transient analysis of laminated beams. Compos. Eng., Int. J. 2: 375-390

Pagano N J 1969 Exact solutions for composite laminates in cylindrical bending. J. Compos. Mater. 3: 398-411

Petersen B R 1985 Finite element analysis of composite plate impacted by a projectile. Ph D dissertation, University of Florida

Pintado P, Vogler T J, Morton J 1991 Impact damage development in thick composite laminates. Compos. Eng. 1: 195-221

Qian Y, Swanson S R 1990 Experimental measurements of impact response in carbon/epoxy plates. $A I A A$ J. 28: 1069-1074

Ramkumar R L, Chen P C 1983 Low-velocity impact response of laminated plates. AIAA J. 21: $1448-1452$

Reddy J N 1980 A penalty plate bending element for the analysis of laminated anisotropic composite plates. Int. J. Numer. Methods Eng. 15: 1187-206

Reddy J N 1983 Geometrically nonlinear transient analysis of laminated composite plates. AIAA J. 21: 621-629

Reddy J N 1987 A generalization of two-dimensional theories of laminated composite plates. Commun. Appl. Numer. Methods 3: 173-180

Reddy J N 1990 A general third-order nonlinear theory of plates with moderate thickness. $J$. Nonlinear Mech. 25: 677-686

Sankar B V, Sun C T 1985a Efficient numerical algorithm for transverse impact problems. Comput. Struct. 20: 1009-1012

Sankar B V, Sun C T 1985b Low velocity impact response of laminated beams subjected to initial stresses. AIAA J. 23: 1962-1969

Sun C T 1977 An analytical method for evaluation of impact damage energy of laminated composites. Am. Soc. Test. Mater., Spec. Tech. Publ. 617: 427-440

Sun C T, Chattopadhyay S 1975 Dynamic response of anisotropic laminated plates under initial stress for impact of a mass. J. Appl. Mech., ASME 97: 693-698

Sun C T, Chen J K 1985 On the impact of initially stressed composite laminates. J. Compos. Mater. 19: 490-504

Sun C T, Liou W J 1989 Investigation of laminated composite plates under impact dynamic loading using a three-dimensional hybrid stress finite element method. Comput. Struct. 33: 879-884 
Swanson S R, Smith N L, Qian Y 1991 Analytical and experimental strain response in impact of composite cylinders. Compos. Struct. 18: 75-108

Tan T M, Sun C T 1982 Wave propagation in graphite/epoxy laminates due to impact. NASA CR-168057

Timoshenko S P 1913 Zur Frage Nach der Wirkung Eines Stosses auf Einen Balken. Z. Math. Phys. 62: 198-209

Timoshenko S, Goodier J N 1970 Theory of elasticity (New York: McGraw-Hill)

Thangiitham S, Librescu L, Cederbaum G 1987 Low-velocity impact response of orthotropic plates using a higher-order theory. Proc. of $28 t h$ AIAA/ASME/ASCE/-AHS/ASC Structures, Structural Dynamics and Materials Conference, pp. 448-457

Yang S H, Sun C T 1981 Indentation law for composite laminates. NASA CR-65460 